\title{
Angiostrongylus vasorum
}

\author{
(Baillet, 1866)
}

\section{Etude biologique et morphologique}

\author{
par Jean GUILHON et Bernadette CENS \\ Laboratoire de Parasitologie, Ecole nationale vétérinaire d'Alfort, \\ F 94700 Maisons-Alfort
}

\begin{abstract}
Résumé
Angiostrongylus vasorum (Baillet, 1866) trouvé dans l'appareil circulatoire d'un chien, à Toulouse (France), par Serres (1853) a été décrit par Baillet (1862-1866).

Son cycle évolutif est resté inconnu jusqu'en 1960. A cette époque l'un d'entre nous a montré qu'une limace (Arion rufus) pouvait jouer le rôle d'hôte intermédiaire. Plusieurs autres mollusques gastéropodes pulmonés (11 sur 17 utilisés) se sont révélés plus ou moins réceptifs.

L'évolution larvaire $\left(\mathrm{L}_{1}-\mathrm{L}_{2}-\mathrm{L}_{3}\right)$ d'Angiostrongylus vasorum, dans le corps du mollusque, dure environ 16 à 18 jours à $23-26^{\circ}$ Celsius. Dans l'organisme du chien, les larves migratrices restent 4-5 jours dans les ganglions lymphatiques abdominaux où s'effectuent les dernières mues dont sont issus les stades $\mathrm{L}_{4}$ et $\mathrm{L}_{5}$. Les immatures empruntent ensuite le système porte $\left(8^{e}\right.$ jour $)$ pour aboutir au ventricule droit et à l'artère pulmonaire $\left(9-10^{\circ}\right.$ jours). Les larves $\mathrm{L}_{1}$ apparaissent dans les fèces 38 à 60 jours $(45$ jours en moyenne) après l'infestation expérimentale.

Des précisions sont apportées sur la morphologie des différents stades et plus particulièrement sur celle du quatrième et sur les caractères de l'appareil génital mâle, pourvu d'un gubernaculum dont la présence était discutée.
\end{abstract}




\section{Summary}

\section{Angiostrongylus vasorum (Baillet, 1866). Morpho- logical and biological study.}

Angiostrongylus vasorum (Baillet, 1866) found in dog's circulatory system in Toulouse (France) by Serres (1853) has been described by Baillet (1862-1866).

Its life-cycle remained unknow till 1960. At that time one of us shown that a slug (Arion rufus) could play the part of intermediate host. Many other pulmonate gasteropod molluses (11 out of 17 utilized) revealed themselves more or less receptive.

Larval evolution $\left(\mathrm{L}_{1}-\mathrm{L}_{2}-\mathrm{L}_{3}\right)$ of Angiostrongylus vasorum in mollusc's body lasts about 16 to 18 days at $23-26^{\circ}$ Celsius. In dog's organism, migratory larvae keep 4-5 days in abdominal visceral lymphatic nodes where take place last moults from which stages $\mathrm{L}_{4}$ and $\mathrm{L}_{5}$ are born. Then immatures follow the portal system $\left(8^{\text {th }}\right.$ day) to reach ventricle and pulmonary artery $\left(9^{\text {th }}\right.$ $10^{\text {th }}$ day). $\mathrm{L}_{1}$ larvae appear in the faeces 38 to 50 days (on an average of 45 days) after experimental infestation.

Accuracies are brought about morphology of the different stages and more particulary about that of the fourth one and about the characters of male genital apparatus provided for a gubernaculum which presence was discussed.

Bien qu'Angiostrongylus vasorum, Nématode parasite du ventricule droit et de l'artère pulmonaire du chien ait été observé, pour la première fois, dès 1853, par Serres, à l'Ecole nationale Vétérinaire de Toulouse, puis décrit par Baillet (1862-1866) dans le même établissement, son cycle demeura longtemps inconnu.

Toutefois, Laulanié (1882-1884), Railliet et Cadiot (1892), et enfin Cuillé et Darraspen (1934), montrèrent que les adultes sont localisés dans le ventricule droit et, le plus souvent, dans la lumière de l'artère pulmonaire et de ses ramifications. Après l'accouplement, les femelles pondent des œufs non embryonnés d'où sortent à l'éclosion des larves $\left(\mathrm{L}_{1}\right)$, que l'on retrouve dans les voies respiratoires et dans les fèces après déglutition du mucus. Mais ces auteurs, soit en faisant ingérer des larves $\mathrm{L}_{1}$ provenant d'excréments de chiens atteints d'angiostrongylose, soit en donnant à consommer les poumons de ces derniers à des sujets apparemment indemnes de cette affection, n'obtinrent aucun résultat positif $\left({ }^{*}\right)$.

L'un de nous ayant eu l'occasion, en 1958, de visiter deux élevages canins (teckels et bleus de Gascogne), dans une propriété de Saint-Jean-Pied-de-Port (Pyrénées-

(*) Laulanié a cru cependant observer des résultats positifs, mais il s'agissait vraisemblablement d'animaux infestés naturellement qui n'avaient pas encore éliminé de larves L 1 , lorsqu'ils furent choisis pour être contaminés expérimentalement. 
Atlantiques), fut frappé par l'intensité de l'infestation, plus particulièrement des teckels $(80 \%)$, qui vivaient dans un chenil enherbé, à l'extrémité d'un grand parc, dans les allées duquel les limaces étaient anormalement abondantes. Ayant observé, par ailleurs, des chiots mordiller celles-ci, et certaines d'entre elles se déplacer sur le bord des écuelles renfermant la nourriture des animaux, il est apparu plausible de supposer que ces limaces pouvaient peut-être intervenir comme hôte intermédiaire dans l'évolution jusqu'alors inconnue d'Angiostrongylus vasorum.

Deux années plus tard, en 1960, des limaces (Arion rufus) furent recueillies par la propriétaire, dans le chenil et dans le parc où s'ébattaient les teckels. Avec les fragments de quelques exemplaires reçus, trois chiens d'âges et de sexes différents (4 mois, 20 mois, 3 ans), nés et élevés à Alfort ou dans la Région parisienne, ont été infestés par voie orale. Le plus jeune a succombé à une virose (maladie de Carré), 14 jours après l'absorption du repas contaminant. Les deux autres sujets sont morts d'angiostrongylose expérimentale. Le mâle de 3 ans $(5,5 \mathrm{~kg})$, dont le ventricule droit et l'artère pulmonaire contenaient 289 parasites adultes, a résisté 48 jours; la femelle de 20 mois $(13 \mathrm{~kg})$, qui en renfermait 1.821 , a vécu 55 jours.

La preuve du rôle des limaces (Arion rufus) dans l'accomplissement du cycle évolutif d'Angiostrongylus vasorum, dans la nature, était ainsi apportée.

La mort, aussi démonstrative que rapide, des sujets utilisés, nous contraignit à renouveler cette expérience princeps aussi bien pour la confirmer que pour posséder un animal donneur permanent.

Un an plus tard, en 1961, une seule chienne, âgée de 7 ans environ, qui a été conservée grâce à une médication symptomatique pendant 10 années (1961-1970), fut infestée par ingestion forcée de quelques fragments de limaces (Arion rufus) provenant du même chenil. Des larves $\mathrm{L}_{1}$ apparurent dans les excréments, 50 jours après l'intervention.

C'est à partir de cette chienne, qui a résisté à une seule et légère infestation, qu'il a été possible d'entretenir une souche au laboratoire et d'entreprendre des recherches sur les diverses phases du cycle évolutif d'Angiostrongylus vasorum.

Le matériel et les méthodes utilisés, ainsi que les résultats obtenus, sont indiqués dans les deux chapitres ci-après :

I. Etude de l'évolution larvaire du parasite dans l'organisme des mollusques;

II. Etude de ses migrations, chez le chien, depuis la contamination par les larves infestantes $\mathrm{L}_{3}$ jusqu'à l'expulsion des larves $\mathrm{L}_{1}$, dans le milieu extérieur, avec les fèces.

\section{Etude de l'évolution larvaire du parasite dans l'organisme des mollusques.}

\section{A) Evolution dans Arion rufus (LinNe).}

Des limaces (Arion rufus), maintenues aux températures de 18 à $23{ }^{\circ} \mathrm{C}$ et à un degré hygrométrique suffisamment élevé, furent infestées par un nombre connu de larves $L_{1}$, provenant de fèces de la chienne contaminée expérimentalement. Elles furent ensuite disséquées et examinées en section mince, sous le microscope. Les larves gran- 
dissent assez rapidement durant les premiers jours de leur évolution intra-tissulaire, et leur mobilité diminue, ensuite, jusqu’à devenir presque imperceptible. Pendant cette période, qui annonce la première mue (vers le $7^{\circ}$ jour), les cellules intestinales se chargent de matières de réserves qui leur confèrent une couleur brunâtre. La plupart des larves $\mathrm{L}_{2}$ apparaissent trois jours plus tard, sombres et trapues, entourées d'une réaction tissulaire de l'hôte; peu mobiles, elles n'effectuent que des déplacements très limités dans leur première peau de mue. Les jours suivants, les réserves diminuent, les larves s'éclaircissent et, dans leur région antérieure, se dessinent deux baguettes chitinoïdes qui se terminent chacune, vers l'avant, par une petite formation subtriangulaire, en arrière de l'orifice buccal. Vers les quinzième et seizième jours, on peut observer les larves, au troisième stade, dans leurs deux fourreaux cuticulaires ; elles deviennent alors de plus en plus transparentes, et les baguettes céphaliques se distinguent nettement. A ce stade, les larves sont plus minces et leur longueur atteint environ $560 \mu$, l'extrémité antérieure est arrondie, alors que la queue est légèrement ondulée.

B) Evolution, dans L'organisme, de Quelques mollusques terrestres ou DULÇAQUiCOLES.

Il a été démontré que non seulement les limaces de la famille des Arionidés, mais aussi de nombreuses espèces de mollusques terrestres ou dulçaquicoles peuvent servir d'hôte intermédiaire à l'égard d' $A$. vasorum. Parmi 17 espèces de mollusques terrestres testées, 11 d'entre elles permettent d'obtenir des larves $\mathrm{L}_{3}$ infestantes après une évolution de 17 jours (à $20-23^{\circ} \mathrm{C}$ ) : Arion lusitanicus, A. hortensis, Deroceras reticulatum, Limax flavus, Helix pomatia, H. aspersa, Cepaea nemoralis, Arianta arbustorum, Euparypha pisana, Succinea putris, Cochlodina laminata, et deux espèces d'eau douce d'origine tropicale, Physa $s p$. et Biomphalaria glabrata, se sont révélées également réceptives.

Dans tous les cas, l'évolution est très semblable à celle observée chez Arion rufus, aux mêmes conditions de températures.

C) Evolution dans Biomphalaria glabrata (SAY).

Ce mollusque dulçaquicole des régions tropicales (Gastéropode pulmoné de la famille des Planorbidés) fut régulièrement utilisé pour conserver plus facilement la souche d'Angiostrongylus vasorum au laboratoire.

Les Planorbes nourries avec des feuilles de laitue vivent dans des bacs en verre (10 litres) contenant de l'eau de source constamment filtrée et maintenue à $25-26^{\circ} \mathrm{C}$, grâce à des résistances électriques.

L'infestation des mollusques est acquise par leur coexistence, pendant une durée variant de 4 à 20 heures, dans de petits cristallisoirs, avec un nombre connu de larves $\mathrm{L}_{1}$ d'Angiostrongylus vasorum, extraites des fèces de chiens par la méthode de Baermann. Le contact est répété plusieurs jours de suite. Les Planorbes contaminées sont ensuite placées dans un bac d'où elles sont prélevées journellement, pour suivre 
l'évolution larvaire, au microscope, après dissection ou digestion peptique de leurs tissus pendant deux heures à $37^{\circ}$, dans une solution contenant $5,5 \mathrm{~g}$ de pepsine, $5 \mathrm{ml}$ d' $\mathrm{HCl}, 1.000 \mathrm{ml}$ d'eau distillée.

\section{$1^{\circ}$ Phases évolutives et principales caractéristiques des transformations larvaires.}

Un nombre variable (3.000 à 10.000) de larves $\mathrm{L}_{1}$ mises en présence de chaque Planorbe sont retrouvées, dans les muscles, 24 heures après l'infestation expérimentale, à un taux très faible (10 à $20 \%$ ). Ces larves, sans manifester un tropisme particulier, sont absorbées semble-t-il avec les aliments. Dès le début de leur séjour dans le mollusque, elles s'enroulent dans les muscles, plus particulièrement dans la masse pédieuse et dans le manteau tapissant la cavité pulmonaire. Cependant, lors d'infestations intenses, elles sont visibles dans presque tous les organes (hépato-pancréas, glande hermaphrodite...) ; nous ne les avons jamais trouvées dans les tentacules, ni dans le gésier. Elles s'immobilisent quelques jours après l'invasion et croissent rapidement, pour passer de $365 \mu \times 16 \mu$, à $450 \mu \times 30 \mu$, en trois à quatre jours. Pendant la même période, des matières de réserves apparaissent dans les cellules intestinales, visibles à travers la cuticule, sous forme de granulations brunâtres.

$\mathrm{Au} 7^{\circ}$ ou $8^{\circ}$ jour de leur évolution, il est possible de distinguer, sur certaines d'entre elles, la première peau de mue qui les enveloppe. Elles ont atteint le deuxième âge, restent incluses dans la cuticule du stade précédent et paraissent emprisonnées dans une réaction tissulaire de leur hôte. Les réserves ont envahi la région intestinale et les larves deviennent plus trapues et noirâtres.

Plus tard, vers le $9^{\circ}$ jour après leur pénétration, elles se mobilisent, s'agitent dans leur première exuvie et effectuent leur deuxième mue.

Enfin, après 16 jours de vie intratissulaire, les réserves sont en général épuisées, les cellules intestinales s'éclaircissent et les larves deviennent transparentes. De fines baguettes chitinoïdes se dessinent nettement à l'extrémité antérieure de l'œsophage. Il est possible, dans des cas favorables, d'observer les deux peaux de mue constitutives de leur fourreau exuvial.

En résumé, la larve $\mathrm{L}_{3}$ est obtenue en 16 à 18 jours, après deux mues, qui se manifestent entre le $7^{\circ}$ et le $8^{\circ}$ jour et entre le $9^{\circ}$ et le $11^{\circ}$ jour après la pénétration des larves $\mathrm{L}_{1}$ dans le corps du mollusque.

\section{$2^{\circ}$ Morphologie des trois premiers stades larvaires.}

a) Premier stade (larve $\mathrm{L}_{1}$ ) (fig. 1).

Les larves du premier âge $\left(\mathrm{L}_{1}\right)$, sorties de l'œuf après éclosion dans la lumière du réseau artériel pulmonaire, s'observent dans l'artère, les artérioles et les capillaires pulmonaires, puis dans le mucus alvéolaire, bronchique et trachéal et, enfin, mélangées aux fèces des chiens avec lesquelles elles sont éliminées à l'extérieur (*).

(*) Dans certaines circonstances exceptionnelles, elles peuvent être entraînées dans le courant circulatoire et envahir diverses régions de l'organisme et, notamment, les méninges (observation faite 3 fois lors d'infestations massives). 
Les larves $\mathrm{L}_{1}$, éléments exclusifs de dissémination du parasite, sont graciles et transparentes. Elles mesurent en moyenne $365 \mu$ de longueur et leur diamètre est d'environ $16 \mu$. Leur extrémité antérieure est arrondie, l'œsophage de type strongyloïde mesure environ $150 \mu$ de longueur. Il est étroit et légèrement renflé dans sa portion terminale; la limite œsophago-intestinale est difficile à distinguer. La région postérieure de la larve s'effile en une queue caractéristique ornée d'unne entaille dorsale, d'une encoche ventrale et se termine par une courte ondulation $(8 \mu)$.

L'aspect de l'extrémité caudale des larves $\mathrm{L}_{1}$ est à souligner, car il constitue un caractère de diagnose permettant leur différenciation de celles des autres Angiostrongles et de celles de divers Métastrongylidés.

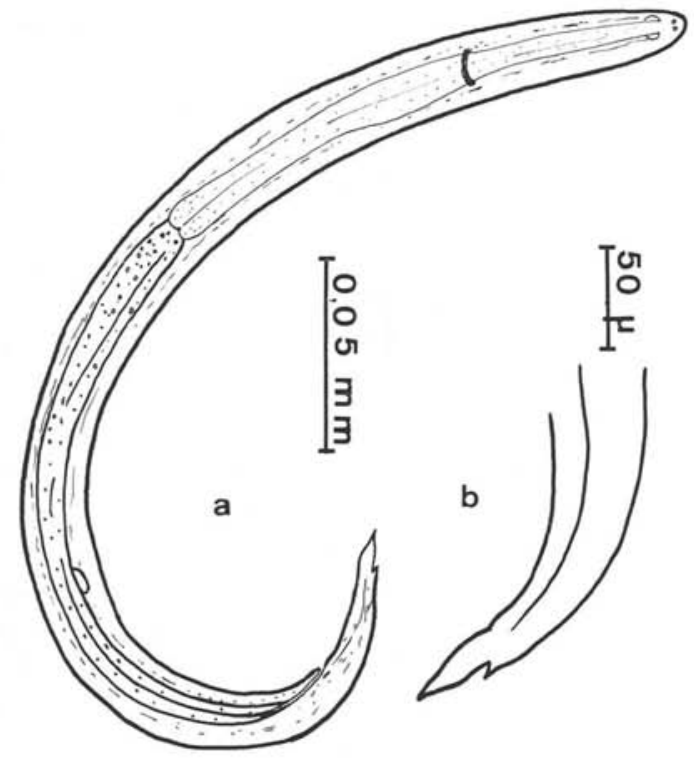

FIg. 1. - Larve L, prélevée dans les fèces d'un chien infesté expérimentalement: $a$ ) vue d'ensemble; $b$ ) détail de l'extrémité caudale.

L'anneau nerveux, très difficile à percevoir, même sur le vivant, est situé à environ $57 \mu$ de l'orifice buccal, près duquel il est parfois possible de constater la présence de deux petites taches réfringentes, dont la signification reste à définir (peut-être marquent-elles l'emplacement des futures formations céphaliques des larves du troisième stade ?).

L'anus s'ouvre ventralement à environ $33 \mu$ de la partie terminale de la queue ; l'ébauche génitale, de forme ovale, mesure environ $11 \mu$ et se situe contre la face ventrale de l'intestin à $130 \mu$ de l'extrémité distale de la larve. 
Tableau I

Mensurations de la larve $L_{1}$

\begin{tabular}{|c|c|}
\hline \multirow{2}{*}{ Corps } & $\begin{array}{c}365 \mu \\
(340-399)\end{array}$ \\
\hline & $\begin{array}{c}16 \stackrel{\mu}{\mu} \\
(13-17)\end{array}$ \\
\hline 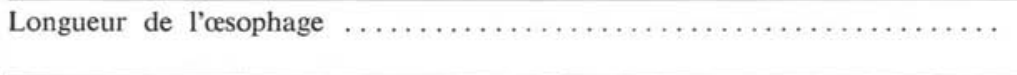 & $\begin{array}{c}150 \mu \\
(144-170)\end{array}$ \\
\hline Distance de l'anneau nerveux à l'extrémité antérieure $\ldots \ldots \ldots \ldots \ldots \ldots$. & $\begin{array}{c}57 \mu \\
(53-61)\end{array}$ \\
\hline 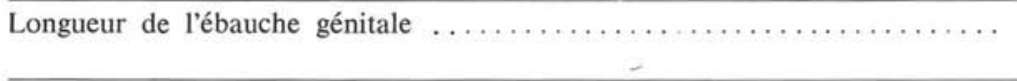 & $\left(\begin{array}{ll}11 \mu \\
(\quad 8-18)\end{array}\right.$ \\
\hline 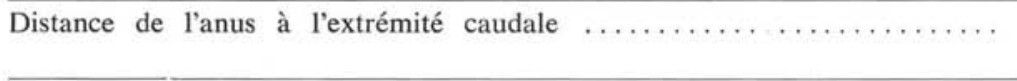 & $\left(\begin{array}{c}33 \mu \\
(31-37)\end{array}\right.$ \\
\hline Longueur de l'ondulation caudale $\ldots \ldots \ldots \ldots \ldots \ldots \ldots \ldots \ldots \ldots \ldots \ldots \ldots \ldots \ldots \ldots \ldots$ & $\begin{array}{c}8 \mu \\
(6-9)\end{array}$ \\
\hline
\end{tabular}

Tableau II

Mensurations de la larve infestante $\mathrm{L}_{3}$

\begin{tabular}{|c|c|c|c|}
\hline \multirow{2}{*}{ Corps } & \multicolumn{3}{|c|}{$\begin{array}{c}555 \mu \\
(528-590)\end{array}$} \\
\hline & \multicolumn{3}{|c|}{$\begin{array}{c}28 \mu \\
(26-33) \\
\end{array}$} \\
\hline Longueur $\ldots \ldots \ldots \ldots \ldots \ldots \ldots \ldots \ldots$ & \multicolumn{3}{|c|}{$\begin{array}{c}201 \mu \\
(185-215)\end{array}$} \\
\hline $\begin{array}{l}\text { Esophage } \\
\text { Diamètre } \ldots \ldots \ldots \ldots \ldots \ldots \ldots \ldots \ldots \ldots\end{array}$ & $\begin{array}{l}\text { antérieur } \\
13 \mu \\
(12-14)\end{array}$ & $\begin{array}{l}\text { milieu } \\
16 \mu \\
(15-18)\end{array}$ & $\begin{array}{l}\text { postérieur } \\
20 \mu \\
(18-22)\end{array}$ \\
\hline 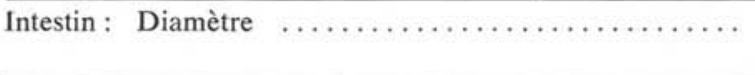 & & $\underset{(15-20)}{18 \mu}$ & \\
\hline Distance du pore excréteur à l'extrémité antérieure .... & & $\begin{array}{c}98 \mu \\
(95-101)\end{array}$ & \\
\hline Distance de l'anneau nerveux à l'extrémité antérieure .. & & $96 \mu$ & \\
\hline Longueur de la gonade $\ldots \ldots \ldots \ldots \ldots \ldots \ldots \ldots \ldots$ & & $\underset{(16-29)}{21 \mu}$ & \\
\hline Distance de l'anus̉ à l'extrémité postérieure $\ldots \ldots \ldots \ldots$ & & $\begin{array}{c}35 \mu \\
(27-43)\end{array}$ & \\
\hline Longueur de l'ondulation caudale $\ldots \ldots \ldots \ldots \ldots \ldots$ & & $\begin{array}{c}8 \mu \\
(8-9)\end{array}$ & \\
\hline Longueur du mucron $\ldots \ldots \ldots \ldots \ldots \ldots \ldots \ldots \ldots \ldots \ldots$ & & $\underset{(1-3)}{2 \mu}$ & \\
\hline
\end{tabular}


b) Deuxième stade (larve $\mathrm{L}_{2}$ ).

La larve du $2^{\circ}$ âge, peu mobile, est le plus souvent disposée en arc de cercle dans une réaction tissulaire de son hôte, enfermée dans le sac exuvial du premier stade qu'elle n'occupe pas entièrement. La présence de granules brunâtres dans les cellules intestinales lui confèrent une couleur marron foncé ou noirâtre. Plus trapue que la précédente elle mesure $420 \mu$ à $450 \mu$ de longueur pour un diamètre de $42 \mu$ environ. Dans la région antérieure

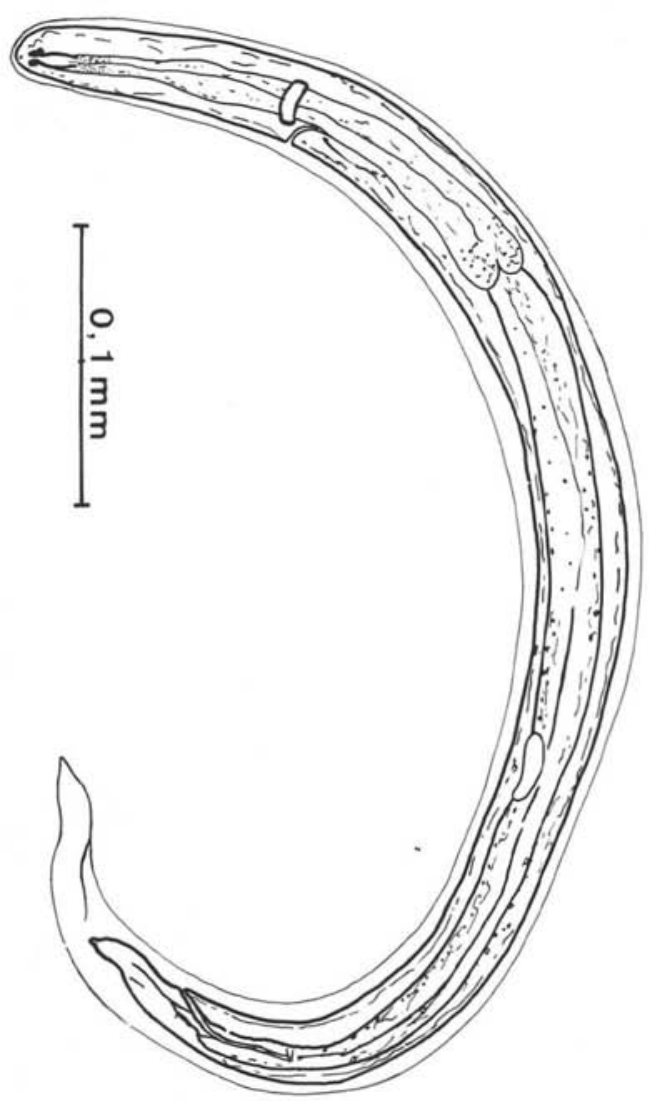
apparaissent des éléments chitinoïdes, parfois incurvés, qui seront plus développés et mieux individualisés au stade suivant. La queue plus courte et pointue rappelle cependant, par son aspect, celle de la larve du premier âge. (fig. 2).

c) Troisième stade (larve $\mathrm{L}_{3}$ )

Le troisième stade larvaire, issu de deux mues se succédant à brefs intervalles, est bien caractérisé par la présence, en région céphalique, d'une paire de baguettes chitinoïdes styliformes (15 à $16 \mu$ ) disposées longitudinalement et ornées antérieurement chacune d'une formation d'aspect le plus souvent triangulaire. La queue s'amincit et se termine par un court appendice digitiforme d'environ $2 \mu$ de longueur. A ce stade, la larve mesure en moyenne $555 \mu$ sur $30 \mu$. Son œsophage, toujours de type stronglyloïde, a environ $200 \mu$ de longueur pour un diamètre de $16 \mu$ dans sa partie moyenne et $20 \mu$ dans sa partie postérieure. Les réserves étant presque entièrement disparues, l'intestin est transparent et débouche sur l'orifice anal situé, ventralement, à environ $35 \mu$ de l'extrémité postérieure. Le pore excréteur, d'où part un fin canal est placé du même côté que l'anus à $98 \mu$ Fig. 2. - Larve $\mathrm{L}_{3}$ extraite de Biomphalaria glabrata. de l'extrémité antérieure. Le collier nerveux péri-œsophagien est situé immédiatement en avant de l'orifice excréteur. Ventralement, contre la paroi intestinale qu'elle repousse, on distingue l'ébauche génitale $(20 \mu)$. La larve est très mobile malgré son fourreau constitué par les deux peaux de mue, que l'on peut différencier plus ou moins nettement en régions céphalique et caudale. 


\section{Evolution dans l'organisme du chien.}

Dans une note antérieure, succincte (1969), l'essentiel des résultats obtenus durant l'étude des migrations dans l'organisme du chien a été publié, mais sans insister sur la description des principaux caractères morphologiques des différents stades évolutifs. Des compléments et des précisions sont fournis dans cette deuxième partie du travail.

Les expériences ont porté sur un effectif de 26 chiens, âgés de 21 jours à 7 mois (45 jours en moyenne), nés et élevés dans l'animalerie du laboratoire, par conséquent à l'abri de toute contamination par les Angiostrongles.

Chaque sujet, à jeun, après tranquillisation au maléate d'acépromazine $(0,5$ à $1 \mathrm{mg} / \mathrm{kg}$ ), reçut, par voie orale, 1.000 à 4.250 larves $\mathrm{L}_{3}$ infestantes, âgées de 25 à 30 jours, incluses dans les fragments tissulaires des Planorbes (Biomphalaria glabrata).

Les chiens furent sacrifiés par injection intraveineuse de sulfate de magnésium à saturation et autopsiés immédiatement après la mort. Pour suivre aussi précisément que possible les diverses phases migratoires des larves $\mathrm{L}_{3}$ ingérées, le premier fut examiné 24 heures après l'infestation et les autres à intervalles réguliers jusqu'à l'apparition des larves $\mathrm{L}_{1}$ dans les fèces.

Les fragments des organes ou tissus prélevés durant l'autopsie furent destinés soit à une étude histologique, soit à l'observation microscopique, après dissection, dans du liquide de Ringer maintenu à $40^{\circ}$.

Enfin, la lymphe, prélevée par exérèse ou cathétérisme du canal thoracique, fut recueillie sur plusieurs sujets aux fins d'examens microscopiques.

Dans ces conditions, il a été possible d'établir une chronologie assez précise des migrations d'Angiostrongylus vasorum dans l'organisme des sujets infestés expérimentalement.

\section{A) Chronologie des migrations dans l'organisme du chien.}

Les larves $\mathrm{L}_{3}$, infestantes, administrées aux chiens, se libèrent de leurs exuvies et traversent activement les parois stomacale et intestinale. Elles sont retrouvées 24 heures plus tard, très mobiles, dépourvues de leur manchon cuticulaire dans les ganglions lymphatiques abdominaux et, plus particulièrement, dans ceux du mésentère.

Le développement larvaire s'effectue dans le tissu ganglionnaire. Trois jours après la contamination, la taille des larves s'est sensiblement accrue $(650-680 \mu \times 30 \mu)$; elles apparaissent plus foncées, et le dimorphisme sexuel est déjà net. Les futurs mâles, plus petits, sont caractérisés par un renflement de la région caudale, où s'ébauche l'appareil copulateur. Chez les femelles, l'orifice vulvaire se forme en position antérieure à celle de l'anus, environ quatre jours après l'infestation, puis le vagin et l'utérus 
apparaissent, tandis que la gonade augmente de volume, en se rapprochant des voies génitales, et se scinde pour donner les deux ovaires, qui s'accroissent vers la région antérieure.

Les baguettes céphaliques caractéristiques du $3^{\circ}$ stade sont expulsées au cours de l'exuviation. Après quatre jours d'évolution, la plupart des larves sont, au quatrième âge, incluses ou non dans leur gaine cuticulaire. Il est relativement facile de les libérer de leur enveloppe, soit par une simple pression sur la lame, soit en créant une différence de pression osmotique du milieu ambiant. Leur taille varie de 720 à $800 \mu$ et atteint 850 à $870 \mu$ vers le cinquième jour.

Le $5^{\circ}$ stade (ou forme juvénile), encore dans les ganglions lymphatiques, apparaît dès le cinquième jour après la contamination. Les femelles immatures ont, en majorité, terminé leur mue, et sont trouvées pour la plupart libres dans le tissu ganglionnaire 5-6 jours après l'infestation, alors que les mâles restent encore enfermés dans leur ancienne cuticule.

Ces faits d'observation montrent que les diverses phases évolutives d'Angiostrongylus vasorum, dans les ganglions mésentériques du chien, se déroulent assez rapidement, puisque toutes les larves qui ont pu se développer arrivent à l'état adulte environ 6 à 7 jours après l'absorption des mollusques contaminés expérimentalement.

Les jeunes « imago », visibles à l'œil nu, mesurent en moyenne 1,3 à $1,5 \mathrm{~mm}$ de longueur. La quatrième et dernière mue terminée, les immatures quittent les ganglions pour envahir le système veineux hépatique, où ils peuvent être observés, en grand nombre, les $8^{\circ}$ et $9^{\circ}$ jours après l'infestation; les plus précoces apparaissent dans le ventricule droit dès le $9^{\circ}$ jour. La lymphe prélevée sur plusieurs chiens, par cathétérisme du canal thoracique ou après son exérèse, se révèle constamment indemne d'Angiostrongles à un quelconque stade évolutif.

Les jeunes Nématodes atteignent le cœur droit puis l'artère pulmonaire, où les premiers sont à demeure environ 10 jours après l'infestation. A leur arrivée dans le système artériel pulmonaire, les femelles, nettement plus nombreuses que les mâles (2/3 de femelles pour $1 / 3$ de mâles), mesurent de 1,9 à $2,1 \mathrm{~mm}$, et ces derniers 1,6 à $1,7 \mathrm{~mm}$.

Vingt jours après la contamination, presque tous les parasites en migration ont gagné leur habitat définitif. Dans l'organisme des femelles, il est possible d'assister parfois à l'ovogénèse grâce à une suffisante transparence de la cuticule. Les ovocytes, jaunâtres, ovales et légèrement biconcaves, s'empilent les uns sur les autres dans les utérus.

Les femelles, dans la proportion d'environ $25 \%$, sont ovigères vers le $33^{\circ}$ jour de l'évolution et, au $36^{\circ}$, elles le sont toutes. Les œufs, brun foncé, ellipsoïdes, à coque mince, progressent dans les voies génitales sans orientation précise. Dans les 24 heures suivantes ils sont pondus et trouvés en grand nombre, en cours de segmentation, dans l'artère pulmonaire et ses nombreuses ramifications. Ils mesurent $80 \mu$, sur $50 \mu$, mais 
peuvent atteindre $100 \mu$ sur $40 \mu$ lorsqu'ils sont embryonnés. Après leur éclosion, de nombreuses larves $\mathrm{L}_{1}$ sont libérées et pénètrent dans la mince paroi des capillaires, traversent l'épithélium alvéolaire et parviennent dans les bronchioles, les bronches et enfin la trachée. Dès lors, elles peuvent être soit expulsées à l'occasion d'une quinte de toux avec le mucus bronchique expectoré, soit, le plus souvent, après déglutition, éliminées à l'extérieur, avec les fèces.

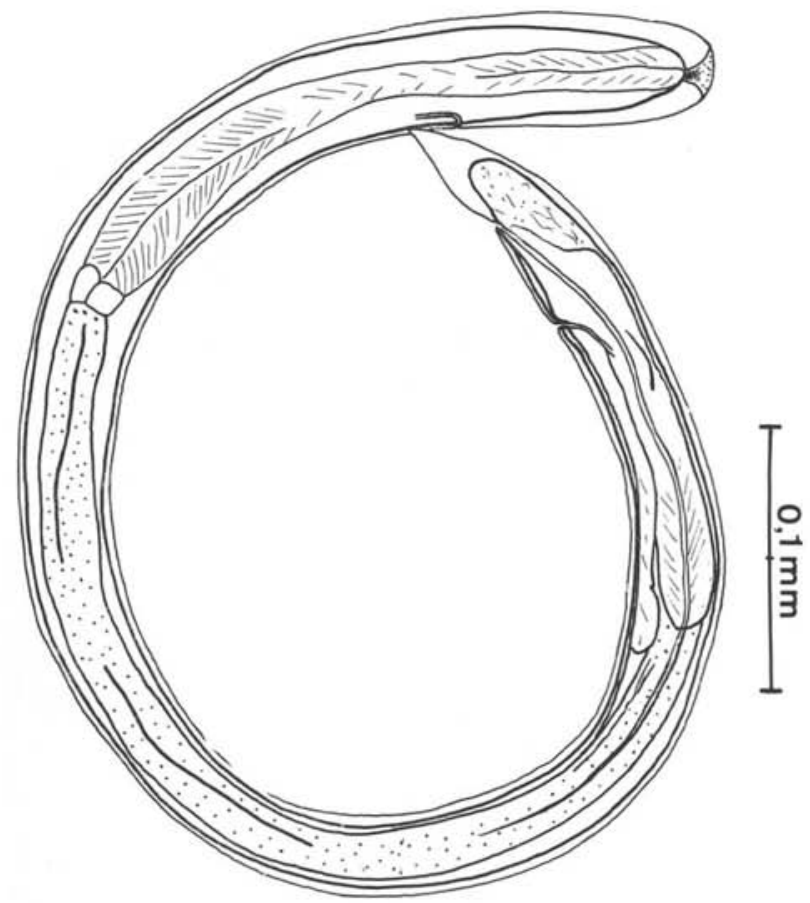

FIG. 3. - Larve $\mathrm{L}_{4}$ extraite d'un ganglion mésentérique de chien, 4 jours après l'infestation.

B) Etude morphologique des larves $\mathrm{L}_{4}, \mathrm{~L}_{5}$, et des adultes.

Les deux derniers stades, qui apparaissent dans les ganglions mésentériques après les $3^{\circ}$ et $4^{\circ}$ mues, sont représentés par les larves $L_{4}$, les immatures et les adultes mâles et femelles $\left(5^{\circ}\right.$ stade).

$1^{\circ}$ Morphologie du quatrième stade larvaire (fig. 3).

Durant la troisième mue, les baguettes chitinoïdes céphaliques du troisième stade sont expulsées et ne sont remplacées par aucun autre organe particulier au niveau de l'orifice buccal. Au début de cette transformation, les larves de sexes différents se 


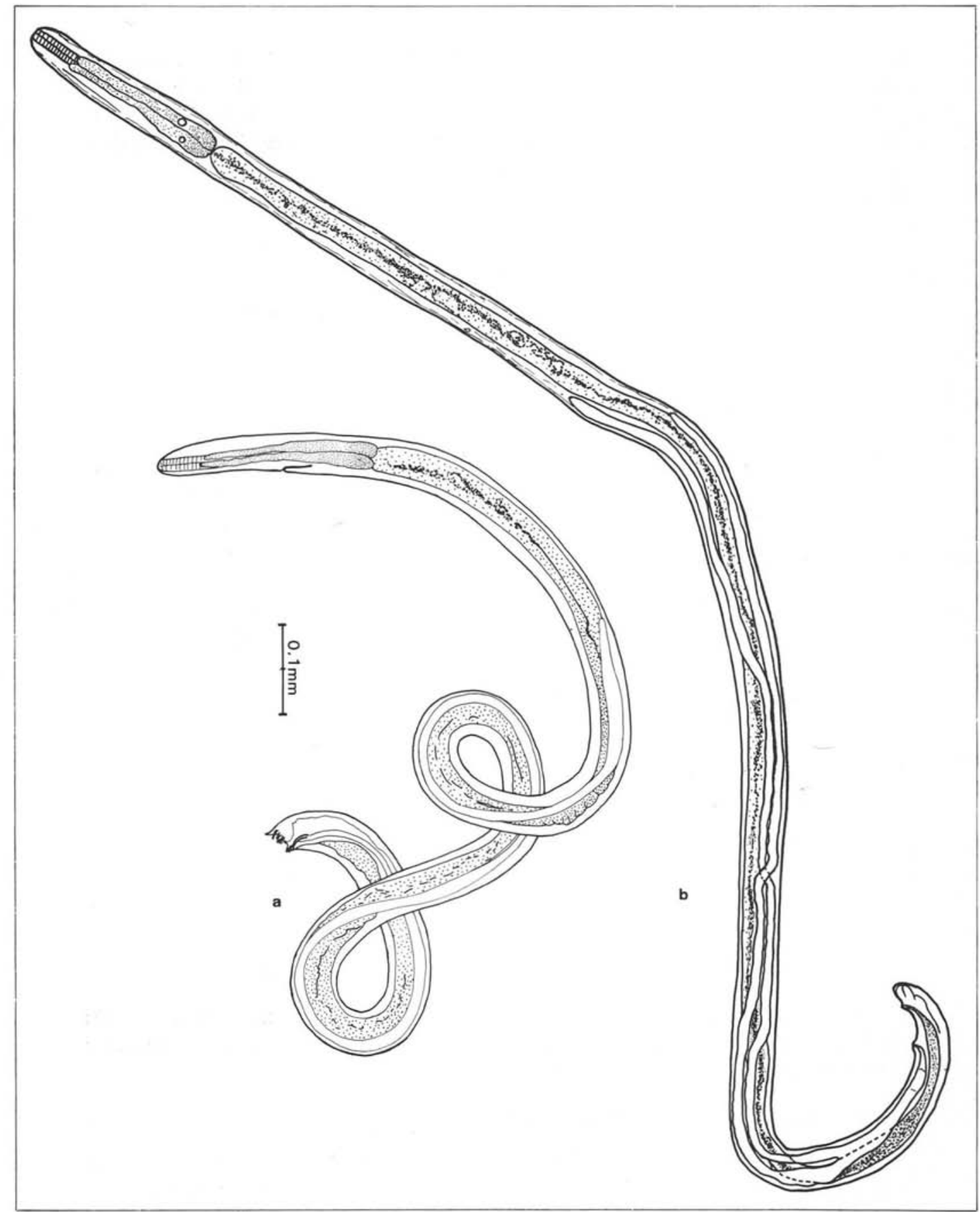

FIG. 4. - Larves $L_{5}$ extraites du foie, 9 jours après l'infestation: a) mâle; b) femelle. 
distinguent nettement par l'aspect de leur queue, qui est renflée surtout chez les futurs mâles. Des remaniements cellulaires annoncent le développement des voies génitales femelles et celui de l'appareil copulateur des mâles.

$\mathrm{Au}$ quatrième stade, difficile à observer du fait de son éphémère existence, le parasite présente une extrémité céphalique arrondie, sans structure particulière. Les jeunes femelles atteignent 700 à $800 \mu$ de longueur ; leur diamètre est sensiblement égal sur toute la longueur du corps (30-35 $\mu$ ). L'œsophage simple, plus musculeux qu'au stade antérieur (larve $\mathrm{L}_{3}$ ), parfois strié, reste toujours clair et mesure $225 \mu$ sur $16 \mu$ environ; sa partie postérieure, plus large, se termine par deux formations plus ou moins arrondies. L'intestin, granuleux et sombre, s'ouvre à l'extérieur par l'anus, situé à environ $20 \mu$ de l'extrémité caudale de la femelle. Placée antérieurement à l'orifice anal $(40-50 \mu)$, la vulve est bien apparente. Le vagin et l'utérus se sont individualisés et rejoignent la gonade, qui se scinde vers la région antérieure et atteint environ $40 \mu$ de longueur chez les plus jeunes larves et $150 \mu$ chez les plus âgées.

Quelques mâles sont pourvus de deux ébauches spiculaires égales mesurant $32,5 \mu$ environ, qui s'organisent au sein d'un amas cellulaire au niveau du rectum. Plus tardivement apparaissent les digitations qui formeront les côtes de la bourse copulatrice du mâle adulte.

Au terme de la quatrième mue, la cuticule est expulsée et, pendant cette opération, les larves augmentent de taille, jusqu'à atteindre environ $1.100 \mu$.

Le quatrième stade donne naissance à des adultes immatures environ six jours après l'infestation de l'hôte définitif.

\section{$2^{\circ}$ Morphologie du cinquième stade.}

Le dernier âge, représenté par les immatures et les adultes arrivés à maturité sexuelle, apparaît après la quatrième et dernière mue.

a) Immatures (fig. 4).

Les jeunes femelles adultes récemment dégagées de leur exuvie, très ténues, mesurent de 1,5 à $2 \mathrm{~mm}$ de longueur sur $50 \mu$ de diamètre.

Les mâles sont constamment plus petits et plus grêles. Tous les organes en place au stade précédent sont généralement plus différenciés. L'œsophage long de 230 à $260 \mu$ est formé de deux parties: une antérieure, musculeuse, claire, striée correspondant à un tiers de son trajet et une autre, granuleuse, plus sombre, d'origine glandulaire occupe les deux tiers postérieurs. Les deux ovaires parallèles des femelles et le testicule des mâles forment des cordons étroits qui s'enroulent autour de l'intestin et dépassent la moitié de la longueur totale du Nématode. La bourse copulatrice du mâle complètement formée reste petite et fragile; les spicules bien apparents sont subégaux. Les jeunes «imago 》 vont s'accroître encore et acquérir leur maturité sexuelle dans leur habitat définitif, environ 33 à 35 jours après l'infestation.

b) Adultes sexués (fig. 5, 6, 7).

La morphologie d'Angiostrongylus vasorum, prototype de la sous-famille des Angiostrongylinés, décrit pour la première fois par Baillet (1866) et étudiée plus récemment par 
L. Rosen et coll. (1970) est connue dans ses grandes lignes (1). Mais, cependant, nous avons cru utile de présenter une nouvelle description de ce Nématode afin d'éviter des confusions possibles avec des espèces voisines, parasites des Canidés.

\section{Tableau III}

Mensurations de l'adulte à maturité sexuelle $\left(^{*}\right)$

\begin{tabular}{|c|c|c|}
\hline \multirow{3}{*}{ Corps } & Femelle & Mâle \\
\hline & $\begin{array}{c}18 \mathrm{~mm} \\
(15-20,5)\end{array}$ & $\begin{array}{c}15 \mathrm{~mm} \\
(14-15,5)\end{array}$ \\
\hline & $\begin{array}{c}270 \mu \\
(220-306)\end{array}$ & $\begin{array}{c}200 \mu \\
(170-235)\end{array}$ \\
\hline \multirow{2}{*}{ CEsophage } & $\underset{(240-280)}{265 \mu}$ & $\stackrel{250 \mu}{(220-275)}$ \\
\hline & $\begin{array}{cc}27 \mu & 40 \mu \\
(23-36) & (34-60)\end{array}$ & $\begin{array}{cc}20 \mu & 33 \mu \\
(14-26) & (26-38)\end{array}$ \\
\hline Diamètre de l'intestin $\ldots \ldots \ldots \ldots \ldots \ldots$ & $\begin{array}{c}185 \mu \\
(130-225)\end{array}$ & $\begin{array}{c}130 \mu \\
(100-150)\end{array}$ \\
\hline $\begin{array}{l}\begin{array}{l}\text { Distance du pore excréteur à l'extrémité } \\
\text { antérieure }\end{array} \ldots \ldots \ldots \ldots \ldots \ldots \ldots \ldots \ldots \ldots \ldots \ldots \ldots \ldots \ldots \\
\end{array}$ & $\begin{array}{c}355 \mu \\
(350-370)\end{array}$ & $\begin{array}{c}330 \mu \mu \\
(310-350)\end{array}$ \\
\hline $\begin{array}{l}\text { Distance de l'anneau nerveux à l'extrémité } \\
\text { céphalique } \quad \ldots \ldots \ldots \ldots \ldots \ldots \ldots \ldots \ldots \ldots \ldots \ldots \ldots \ldots\end{array}$ & 80 à $96 \mu$ & 80 à $92 \mu$ \\
\hline Distance de la vulve à l'anus .......... & $\begin{array}{c}180 \mu \\
(150-220)\end{array}$ & \\
\hline Distance de l'anus à l'extrémité postérieure & $\begin{array}{c}78 \mu \\
(67-100)\end{array}$ & \\
\hline 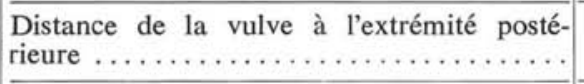 & $\begin{array}{c}250 \mu \\
(220-315)\end{array}$ & \\
\hline $\begin{array}{l}\text { Longueur des spicules } \ldots \ldots \ldots \ldots \ldots \ldots \\
\end{array}$ & & $\begin{array}{cc}450 \prime \prime & 480 \mu \\
(400-480) & (450-500)\end{array}$ \\
\hline Longueur du gubernaculum $\ldots \ldots \ldots \ldots$ & & $\begin{array}{r}46 \mu \\
(40-55)\end{array}$ \\
\hline
\end{tabular}

(*) Les parasites ont été mesurés après fixation et montage (formol Railliet et glycérine gélatinée).

\section{Aspect général.}

Angiostrongylus vasorum (Baillet, 1866) se présente sous l'aspect d'un élément filiforme, nettement visible à l'œil nu.

Les dimensions de la femelle varient de 15 à $21 \mathrm{~mm}$ de longueur sur $220 \mu$ à $306 \mu$ de diamètre en région médiane. Sa teinte, le plus souvent rosée, est due à la présence de

(1) P.C. Gonçalves, en 1961, au Brésil, a donné une description d'Angiostrongylus vasorum à partir d'une femelle et d'un mâle, dont la bourse copulatrice était altérée. 
deux cordons génitaux blancs, enroulés en hélice autour de l'intestin rougeâtre. Cette particularité anatomique est facilement observable grâce à la transparence de la cuticule qui est finement striée transversalement et longitudinalement. L'extrémité postérieure est arrondie et faiblement recourbée du côté ventral.
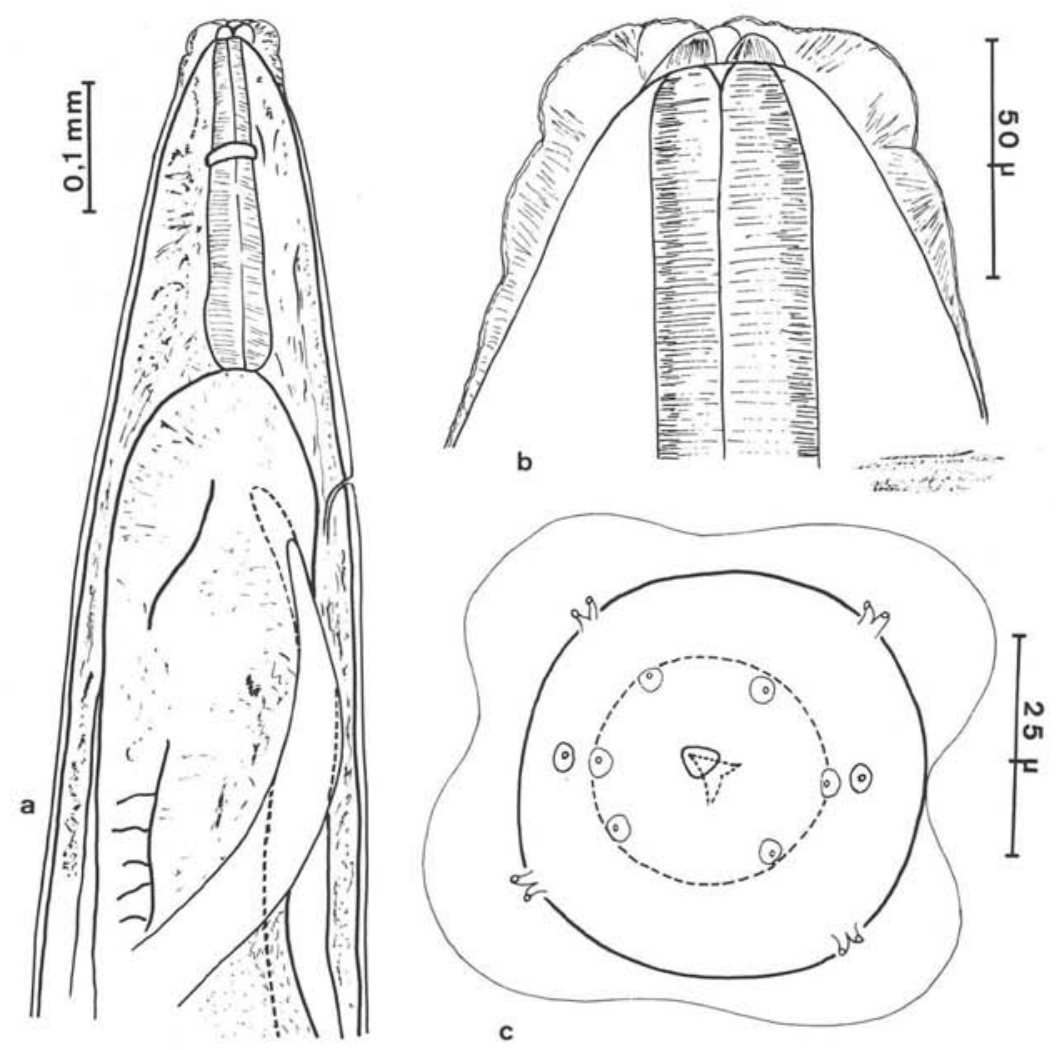

Fig. 5. - Adulte femelle: $a$ ) région antérieure; $b$ ) détail de l'extrémité antérieure ; c) vue apicale.

Le mâle, plus petit (14 à $16 \mathrm{~mm})$ et plus grêle $(170 \mu$ à $230 \mu)$ est blanchâtre ; son extrémité caudale est généralement recourbée ventralement en forme de crosse.

Dans certains cas favorables on peut observer à l'extrémité antérieure des $\sigma^{0}$ et des 우 une gaine tégumentaire, plus ou moins turgescente, formant 4 lobes submédians.

\section{Appareil digestif.}

La bouche, terminale, de section subtriangulaire est entourée, semble-t-il, de 3 lèvres difficiles à voir. Les organes sensoriels en vue apicale sont répartis comme ci-après :

6 papilles labiales internes submédianes; 
2 amphides latérales;

4 papilles labiales externes accolées aux 4 papilles céphaliques, toutes pédonculées.
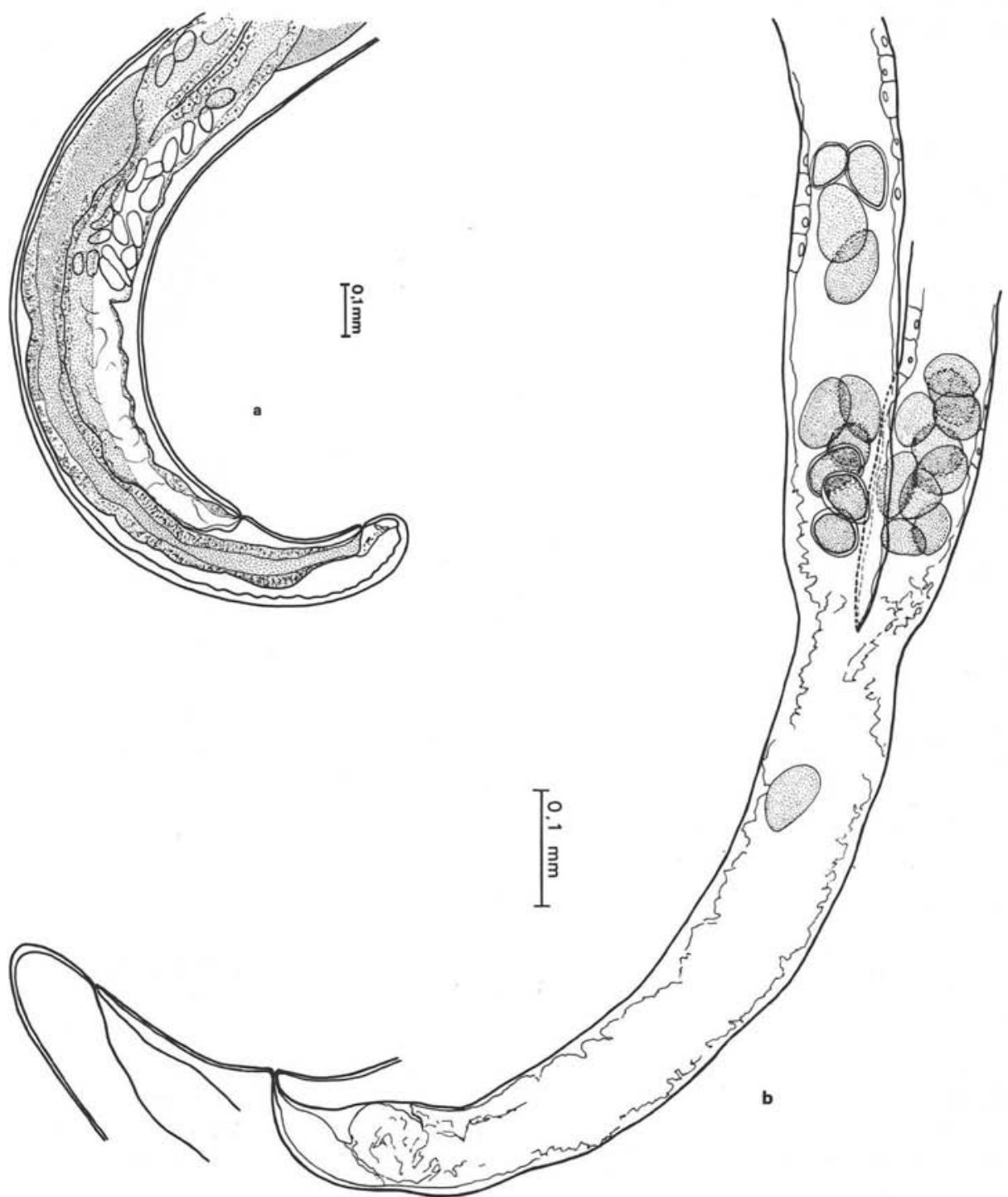

Fig. 6. - Adulte femelle: $a$ ) extrémité postérieure; $b$ ) voies génitales disséquées.

L'orifice buccal donne directement accès à un œsophage court $(220 \mu$ à $280 \mu)$, étroit ( $30 \mu$ à $35 \mu$ en son milieu) de section triradiaire, renflé à son extrémité distale et ne paraissant point morphologiquement différencié en 2 parties. 
L'intestin, long, sinueux, parfois avec des replis, est constamment entouré par les cordons génitaux. Son diamètre $(130 \mu$ à $185 \mu)$ est nettement supérieur à celui de l'œsophage. L'anus subterminal est situé, en moyenne, à $80 \mu$ de l'extrémité caudale de la femelle et à environ $50 \mu$ de celle du mâle.

\section{Appareil reproducteur.}

\section{- Femelle (fig. 6).}

Les ovaires, longs et cylindriques, prennent naissance en arrière de l'œsophage et restent sensiblement contigus dans leur enroulement hélicoïdal autour de l'intestin. Ils se prolongent chacun par un oviducte, étroit canal circonvolutionné, qui donne accès à un utérus très large, le plus souvent rempli d'ovocytes. Ces tubes pairs confluent pour former un vagin qui s'ouvre à l'extérieur par la vulve, située antérieurement à l'anus dont elle est distante de $180 \mu$ environ. L'ovéjecteur est formé de 2 parties: le vestibule et la trompe; le sphincter semble absent. A proximité de la vulve, sur une courte longueur, l'ovéjecteur paraît doublé intérieurement par la cuticule. Il présente 2 constrictions, plus ou moins nettes, avec une cloison transversale à leur niveau obturant une partie de sa lumière. Le vestibule forme une cavité à paroi épaisse et donne accès à une trompe musculo-épithéliale en forme d'Y puisqu'elle semble se dédoubler au niveau d'un rétrécissement avant de donner naissance aux utérus. Le segment impair (vestibule et trompe) mesure environ $730 \mu$ à $800 \mu$.

- MAle (fig. 7-8-9).

Le mâle ne possède qu'un seul testicule qui se présente sous l'aspect d'un cordon blanchâtre, légèrement aplati ; il prend naissance au-dessous de l'œsophage et s'enroule autour de l'intestin. Le canal déférent, étroit, débouche au niveau de l'anus subterminal. La bourse copulatrice est bien visible mais peu développée comparativement à celle des Nématodes Strongylata gastro-intestinaux. Elle est décrite, ci-après, selon la nomenclature établie par Loss et Ransom.

La bourse caudale, petite, mais typique, est souvent orientée ventralement. Dépourvue de lobe médian, elle est constituée de deux lobes latéraux presque identiques et symétriques. Ils sont soutenus par six paires de côtes rigides. Les deux ventrales sont soudées sur les $2 / 3$ de leur longueur. Les trois latérales partent d'un tronc commun, mais l'antéro-latérale s'individualise près de l'insertion alors que les deux autres latérales sont séparées sur environ la moitié de leur trajet. Enfin, l'externo-dorsale, plus courte et plus grêle que toutes les autres côtes, prend naissance isolément. La dorsale, de faible longueur, large, en position sensiblement médiane, se termine par deux digitations le plus souvent inégales, la droite généralement de plus grandes dimensions que la gauche.

Les 2 spicules logés chacun dans une gaine sont jaune brunâtre, fortement chitinisés et finement striés transversalement. Ils sont grêles, moyennement développés et subégaux (droit : $450 \mu$; gauche : $486 \mu$ ). Leur extrémité proximale (capitulum) présente un renflement ovalaire, alvéolé et à bords crénelés. Ils sont disposés parallèlement sur la plus grande longueur du calamus pour ne se séparer qu'à leur extrémité distale (lamina) ou au contraire restent constamment distincts l'un de l'autre.

Chaque spicule est pourvu sur sa face interne d'une aile latérale finement striée, dirigée ventralement. En coupe transversale, leur structure apparaît de section subcirculaire. Grâce à la disposition de leurs ailes, ils peuvent former une gouttière favorisant le passage des 
spermatozoïdes. L'extrémité distale semble trifurquée. Au niveau du cloaque il est possible. d'observer, après éclairicissement du parasite, un épaississement chitinoïde de la gaine spiculaire qui forme le gubernaculum de faible dimension $(46 \mu$ de longueur) et difficile à mettre

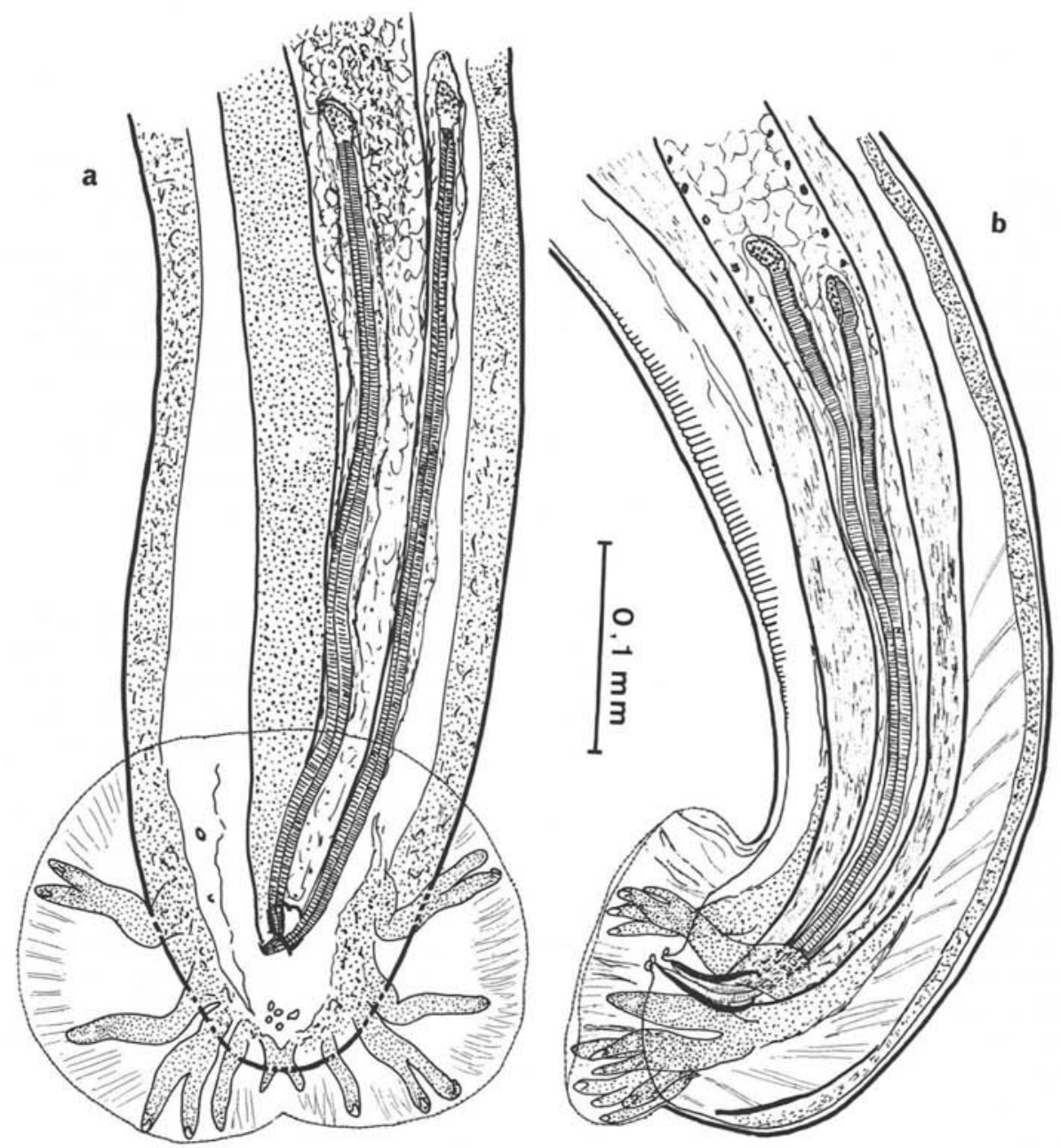

Fig. 7. - Adulte mâle: a) extrémité caudale, vue ventrale; b) extrémité caudale, vue latérale.

en évidence. Il est constitué de 2 éléments latéraux qui guident les spicules dans leur mouvement. Des papilles sensorielles (une précloale impaire et médiane et une paire postcloacale) existent à proximité de l'extrémité distale du gubernaculum. 

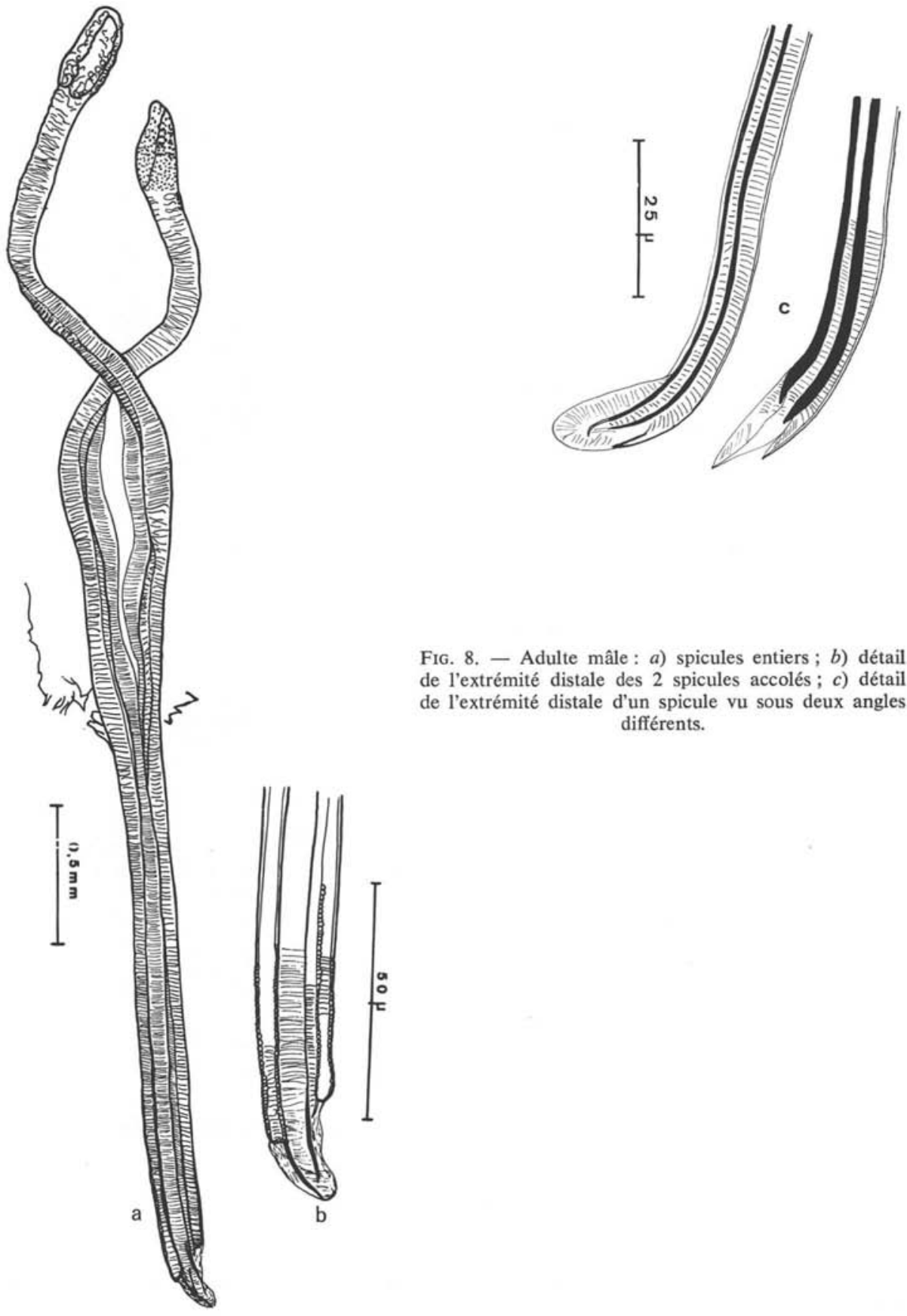

Fig. 8. - Adulte mâle : $a$ ) spicules entiers; $b$ ) détail de l'extrémité distale des 2 spicules accolés; $c$ ) détail de l'extrémité distale d'un spicule vu sous deux angles différents. 


\section{Système excréteur.}

Sur la face ventrale s'ouvre le pore excréteur situé à environ $350 \mu$ de l'extrémité antérieure d'où part, perpendiculairement à la paroi du corps, un fin canal dont la direction s'infléchit vers la région postérieure du parasite.
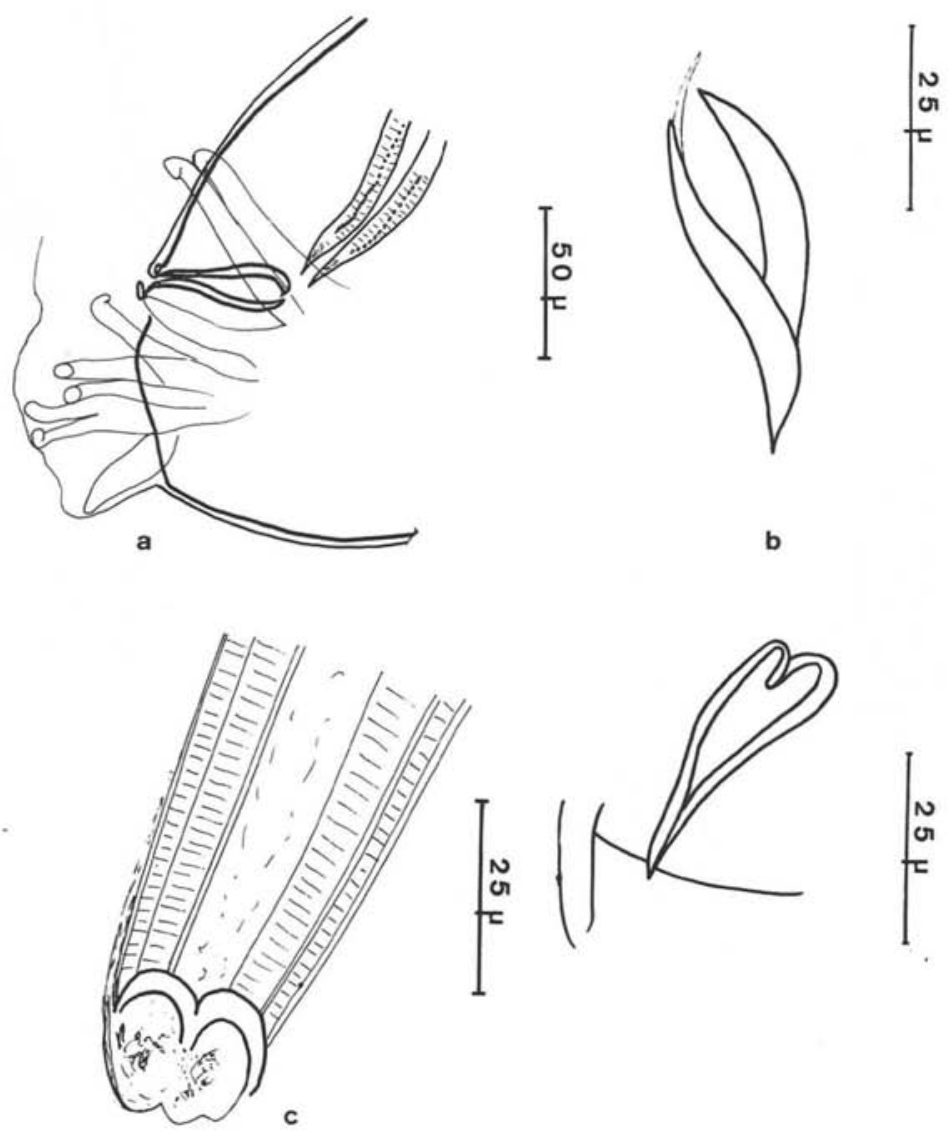

Fig. 9. - Gubernaculum : $a$ ) vue de profil ; $b$ ) deux aspects, face ventrale ; c) vue dorsale.

\section{Système nerveux.}

Sur les adultes vivants on peut observer un anneau péri-œsophagien situé en moyenne à 80-90 $\mu$ de l'extrémité céphalique correspondant environ au tiers antérieur de l'œsophage 


\section{Discussion}

Les résultats des recherches que nous avons entreprises, depuis 1960, pour connaître aussi précisément que possible les diverses phases évolutives d'Angiostron-

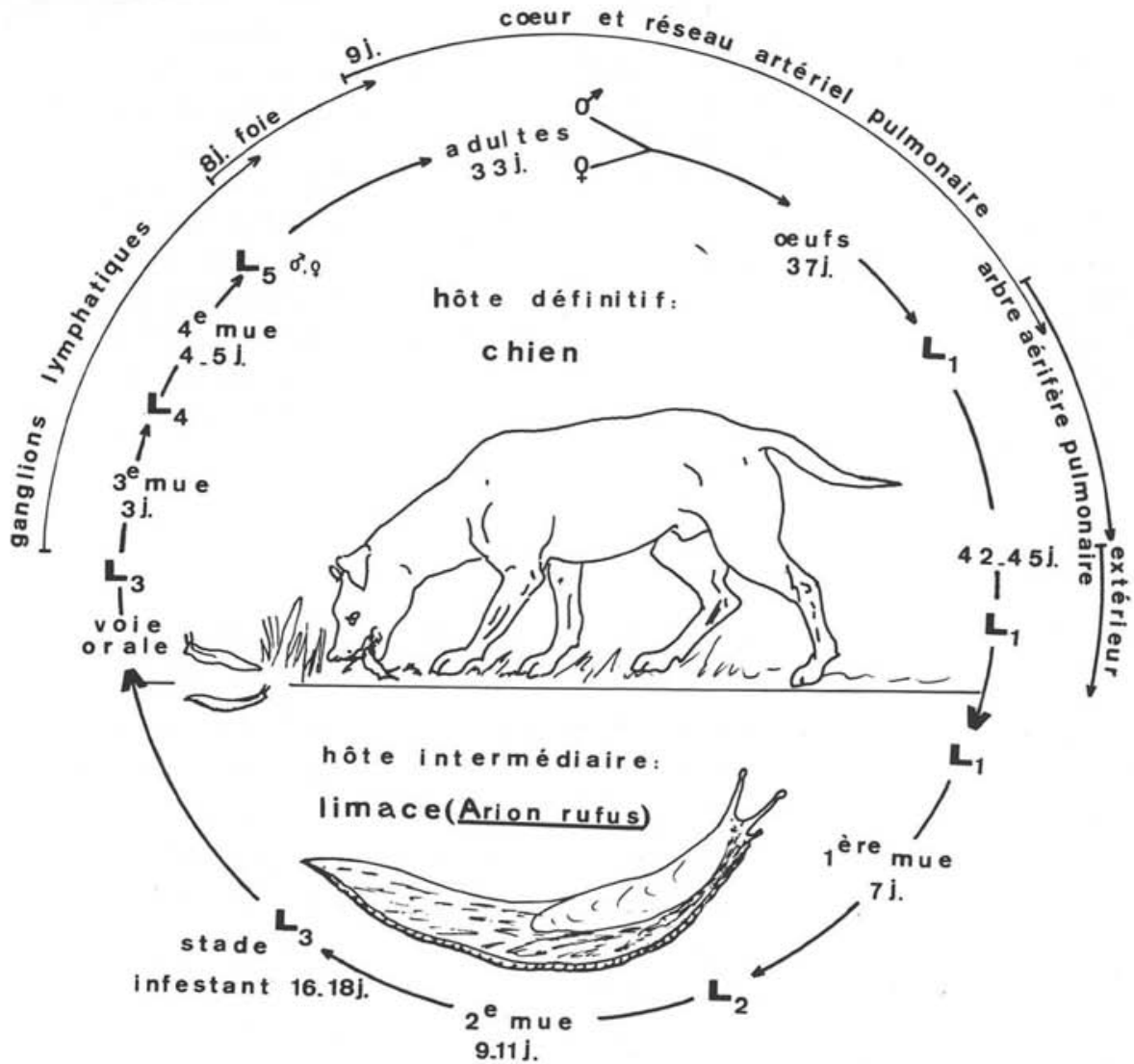

Fig. 10. - Schéma du cycle évolutif d'Angiostrongylus vasorum.

gylus vasorum, ont été récemment confirmées (1970), notamment en ce qui concerne les migrations dans l'organisme du chien, par Rosen, Ash et Wallace aux EtatsUnis (1). Ces auteurs ont constaté comme nous-mêmes que les larves migratrices du parasite ne se dirigent jamais vers les centres nerveux, comme celles d'Angiostrongylus en 1964 .

(1) Avec la souche entretenue au laboratoire, depuis 1960 , que nous avons remise au $\mathrm{D}^{r}$ Rosen, 
cantonensis, qui y séjournent environ 25 à 30 jours avant de gagner l'appareil circulatoire du rat (Mackerras et Sandars). Toutefois, l'observation microscopique de lésions de leptoméningite, avec présence de larves sous la pie-mère, laisse entendre que des troubles nerveux peuvent être provoqués, plus spécialement, lors d'infestation massive par des larves erratiques d'Angiostrongylus vasorum.

Par ailleurs, il convient de souligner que, parmi les nombreux Métastrongylidés dont le cycle évolutif est connu, seul Angiostrongylus vasorum effectue rapidement (4 à 5 jours) ses deux mues dans les ganglions lymphatiques abdominaux du chien, plus particulièrement dans ceux du mésentère, ce qui semble lui conférer une plus grande spécificité. Enfin, si certaines espèces de mollusques gastéropodes terrestres ou même dulçaquicoles sont réceptives et permettent l'évolution larvaire du Nématode dans les conditions expérimentales, ce sont surtout les limaces de la famille des Arionidés, et plus particulièrement Arion rufus, qui paraît être l'hôte le plus fréquemment infesté dans la nature, en France. Cette constatation n'implique pas nécessairement que cette dernière soit l'hôte intermédiaire exclusif ou préférentiel, mais son écologie et son régime omnivore favorisent, vraisemblablement, un contact plus fréquent avec les fèces du chien.

\section{Conclusion}

Les travaux effectués, depuis 1960, pour connaître le cycle évolutif d'Angiostrongylus vasorum, permettent de formuler les conclusions ci-après :

$1^{\circ}$ Angiostrongylus vasorum est un parasite dixène, dont le cycle évolutif comporte obligatoirement, dans les conditions naturelles, le passage dans l'organisme d'un mollusque gastéropode pulmoné ;

$2^{\circ}$ Expérimentalement, l'évolution larvaire se déroule suivant les lois de Maupas, en 16 à 18 jours, entre 23 et $26^{\circ} \mathrm{C}$, dans le corps de plusieurs espèces de mollusques (11 sur 17 utilisées);

$3^{\circ}$ Dans l'organisme du chien, les larves, après un court séjour (4-5 jours), pour assurer leurs deux dernières mues, dans les ganglions mésentériques, empruntent au cinquième stade (immatures) la voie hépatique (dès le $8^{\circ}$ jour), pour aboutir au ventricule droit et surtout dans l'artère pulmonaire et ses nombreuses ramifications $\left(9^{\circ}-10^{\circ}\right.$ jours) ;

$4^{\circ}$ Les adultes atteignent leur maturité sexuelle en 33 à 36 jours ; les œufs sont pondus vers le $37^{\circ}$ jour, et les larves au premier stade apparaissent dans les fèces 38 à 50 jours (en moyenne 45 jours) après l'infestation expérimentale ;

$5^{\circ}$ Des précisions sont apportées sur la morphologie des différents stades larvaires et sur celle des adultes, notamment sur les caractéristiques du quatrième âge et sur les particularités de l'appareil génital mâle, constamment pourvu d'un gubernaculum. 


\section{Tableau IV}

Chronologie des migrations dans l'organisme du chien

\begin{tabular}{|c|c|c|}
\hline $\begin{array}{c}\text { Durée } \\
\text { de l'infestation }\end{array}$ & $\begin{array}{l}\text { Phases évolutives } \\
\text { et migratrices }\end{array}$ & Organes ou Tissus \\
\hline 0 jour $\ldots \ldots \ldots \ldots$ & Infestation & \\
\hline \multicolumn{3}{|c|}{ Arrivée dans les ganglions } \\
\hline 1 jour $\ldots \ldots \ldots \ldots$ & $\mathrm{L}_{3}$ & Gangiions mésentériques. \\
\hline 2 jours $1 / 2 \ldots \ldots \ldots$ & $\mathrm{L}_{3}$ & $\gg$ \\
\hline \multicolumn{3}{|c|}{ Début de la troisième mue } \\
\hline 3 jours ........... & $\mathrm{L}_{3}$ et $\mathrm{L}_{4}$ (peu) & $\gg$ \\
\hline 3 jours $1 / 2 \ldots \ldots \ldots$ & Surtout $\mathrm{L}_{4}$ & $\gg$ \\
\hline 4 jours $\ldots \ldots \ldots \ldots$ & $\mathrm{L}_{4}$ & $\gg$ \\
\hline \multicolumn{3}{|c|}{ Début de la quatrième mue } \\
\hline s jours $\ldots \ldots \ldots \ldots$ & $\begin{array}{l}\mathrm{L}_{4} \text { et } \mathrm{L}_{5} \text { dans ieur peau } \\
\text { de mue. }\end{array}$ & \\
\hline 5 jours $1 / 2 \ldots \ldots \ldots$ & $\begin{array}{l}\text { Immatures: } \\
\mathrm{L}_{\overline{5}} \text { dans leur peau de mue, } \\
\mathrm{L}_{5} \text { libres. }\end{array}$ & $\gg$ \\
\hline 6 jours $\ldots \ldots \ldots \ldots$ & $\begin{array}{l}\mathrm{L}_{5} \text { (avec ou sans peau de } \\
\text { mue). }\end{array}$ & $\gg$ \\
\hline 7 jours $\ldots \ldots \ldots \ldots$ & $\mathrm{L}_{\mathrm{n}}$ libres. & $\gg$ \\
\hline \multicolumn{3}{|c|}{ Arrivée dans le foie } \\
\hline 8 jours $\ldots \ldots \ldots \ldots$ & Jeunes immatures libres. & $\begin{array}{l}\text { Ganglions mésentériques } \\
(++) \text { et foie }(+)\end{array}$ \\
\hline 9 jours $\ldots \ldots \ldots \ldots$ & Immatures. & $\begin{array}{l}\text { Gangiions }(+) \\
\text { Foie }(++)\end{array}$ \\
\hline \multicolumn{3}{|c|}{ Arrivée dans le cœur } \\
\hline 10 jours $\ldots \ldots \ldots \ldots$ & Immatures. & $\begin{array}{l}\text { Ganglions }(+) \\
\text { Foie }(+++) \\
\text { Cour droit, artère et arté- } \\
\text { rioles pulmonaires }(++)\end{array}$ \\
\hline 25 jours $\ldots \ldots \ldots \ldots$ & $\begin{array}{l}\text { Jeunes adultes non encore à } \\
\text { maturité sexuelle. }\end{array}$ & Fines artérioles puimonaires. \\
\hline 27 jours $\ldots \ldots \ldots \ldots$ & $\gg$ & $\begin{array}{l}\text { Cœur et fines artérioles pul- } \\
\text { monaires. }\end{array}$ \\
\hline 29 jours .......... & $\gg \quad \gg$ & $\gg$ \\
\hline \multicolumn{3}{|c|}{ Maturité sexuelle } \\
\hline 33 jours $\ldots \ldots \ldots \ldots$ & $\begin{array}{l}\text { Aduites: } 1 / 4 \text { des femelles } \\
\text { sont fécondées. }\end{array}$ & $\begin{array}{l}\text { Cœur et fines artérioles pul- } \\
\text { monaires. }\end{array}$ \\
\hline 36 jours $\ldots \ldots \ldots \ldots$ & $\begin{array}{l}\text { Toutes les femelles sont } \\
\text { fécondées. }\end{array}$ & $\gg$ \\
\hline 37 jours $\ldots \ldots \ldots \ldots$ & $\begin{array}{l}\text { Euis pondus non segmentés } \\
\text { nombreux. }\end{array}$ & $\begin{array}{l}\text { Fines artérioles pulmonaires, } \\
\text { peu dans le cœur. }\end{array}$ \\
\hline $\begin{array}{l}40 \text { jours } \ldots \ldots \ldots \ldots \ldots \\
38 \text { jours } \ldots \ldots \ldots \ldots\end{array}$ & $\begin{array}{l}\text { Emission des premières lar- } \\
\text { ves } L_{1} \text {. }\end{array}$ & $\longrightarrow$ Fèces. \\
\hline
\end{tabular}



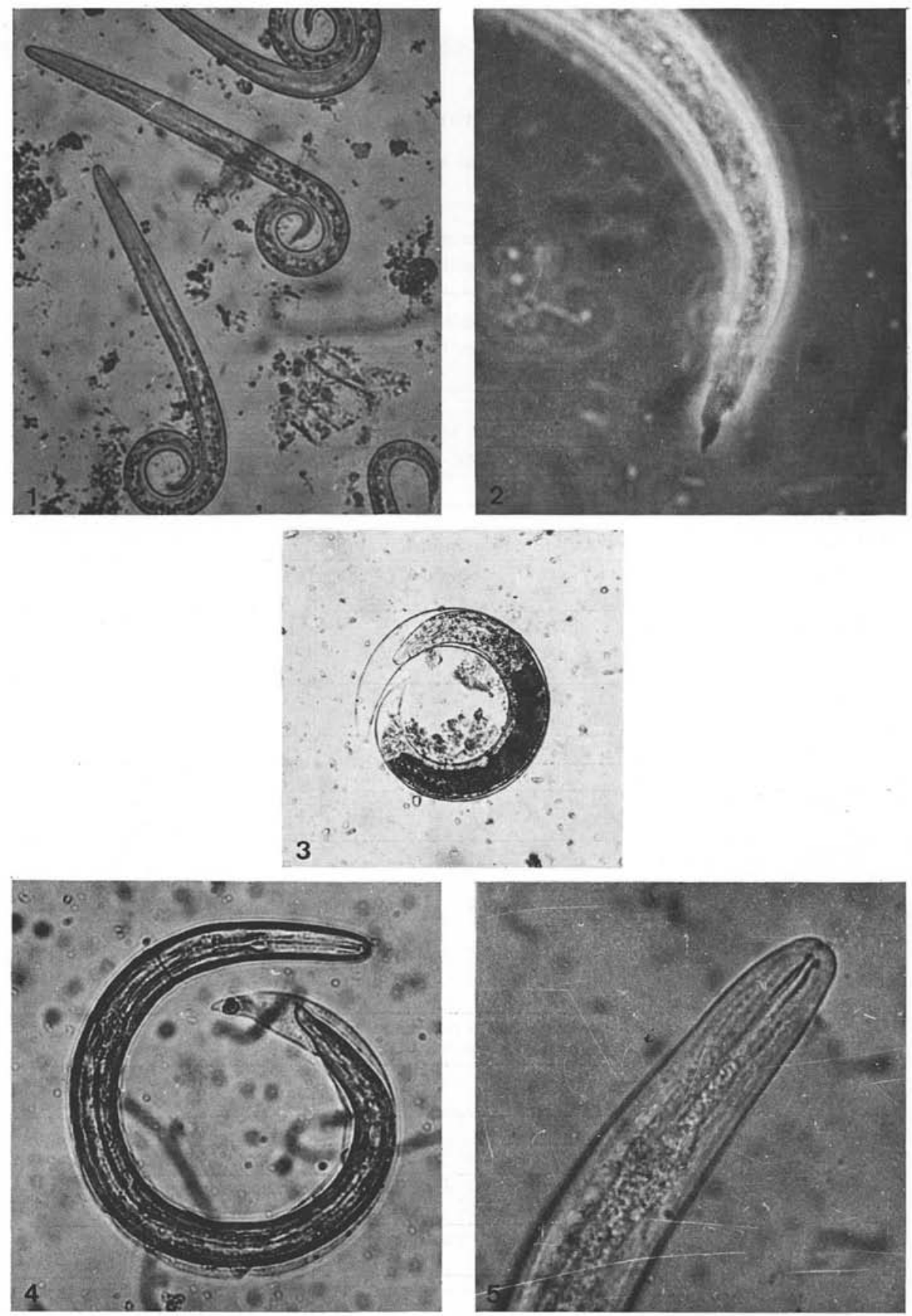

Planche I

Рното 1. - Larves au premier stade prélevées dans les fèces $(\times 125)$. Рното 2. - Extrémité caudale d'une larve $\mathrm{L}_{1}(\times 500)$. Рното 3 . - Larve au deuxième âge extraite d'une Limace $(\times 80)$. Pното 4. - Larve au troisième stade, dans l'exuvie, extraite de Biomphalaria glabrata ( $\times 125)$. Рното 5. - Extrémité antérieure d'une larve $\mathrm{L}_{3}(\times 320)$. 

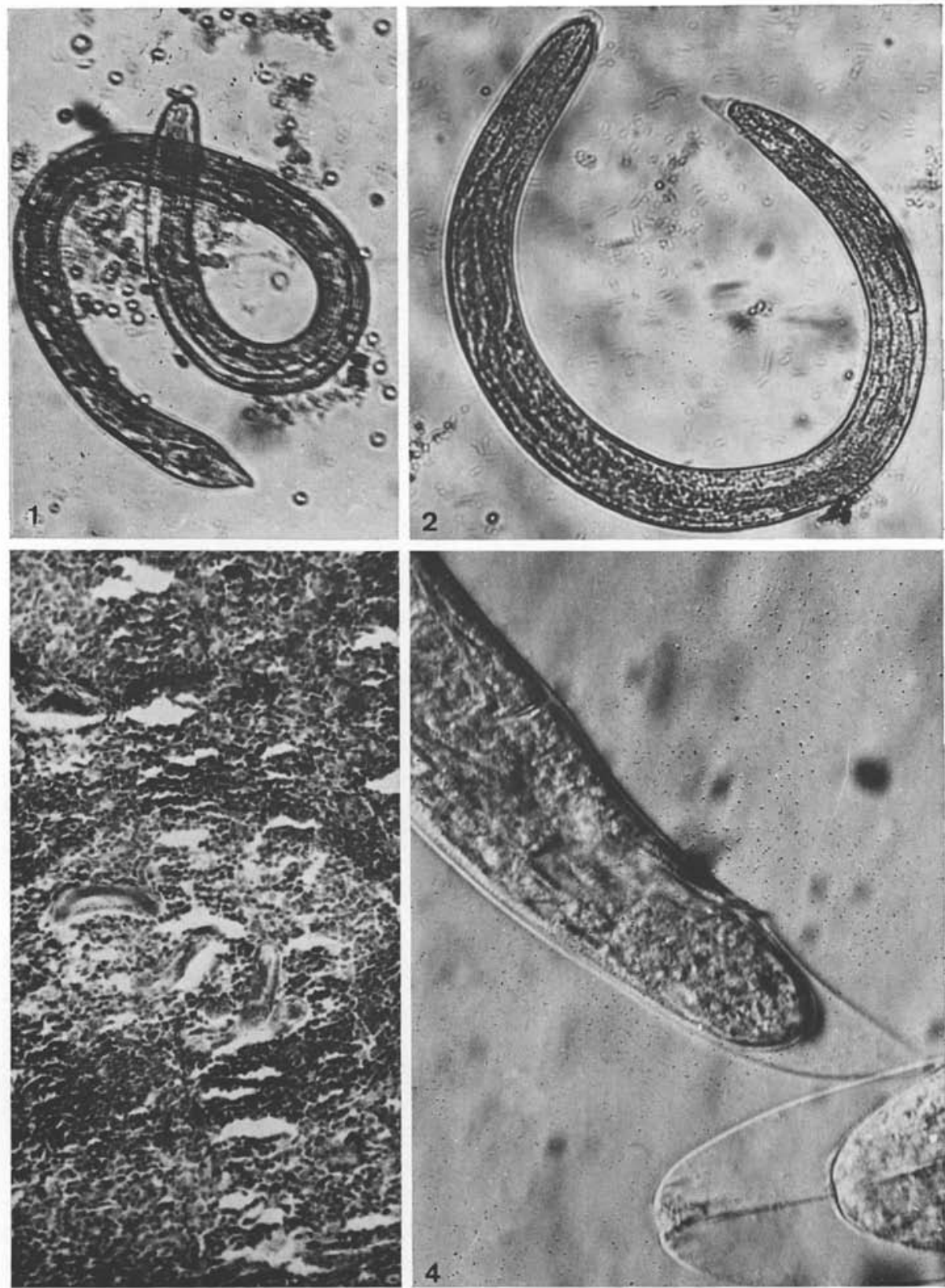

Planche II

Рното 1. - Larve $\mathbf{L}_{3}$, au début de la $3^{e}$ mue, trouvée dans un ganglion mésentérique 3 jours après l'infestation $(\times 125)$. Рното 2. - Larve $\mathrm{L}_{4}$, dans son exuvie, trouvée dans un ganglion mésentérique 4 jours après l'infestation $(\times 125)$. Рното 3. - Larve $\mathrm{L}_{s}$, en coupe, dans un follicule de ganglion mésentérique 4 jours après l'infestation $(\times 80)$. Рното 4 . - Extrémités antérieure et caudale d'une future femelle, dans son exuvie, trouvée 4 jours après l'infestation dans un ganglion mésentérique $(\times 500)$. 

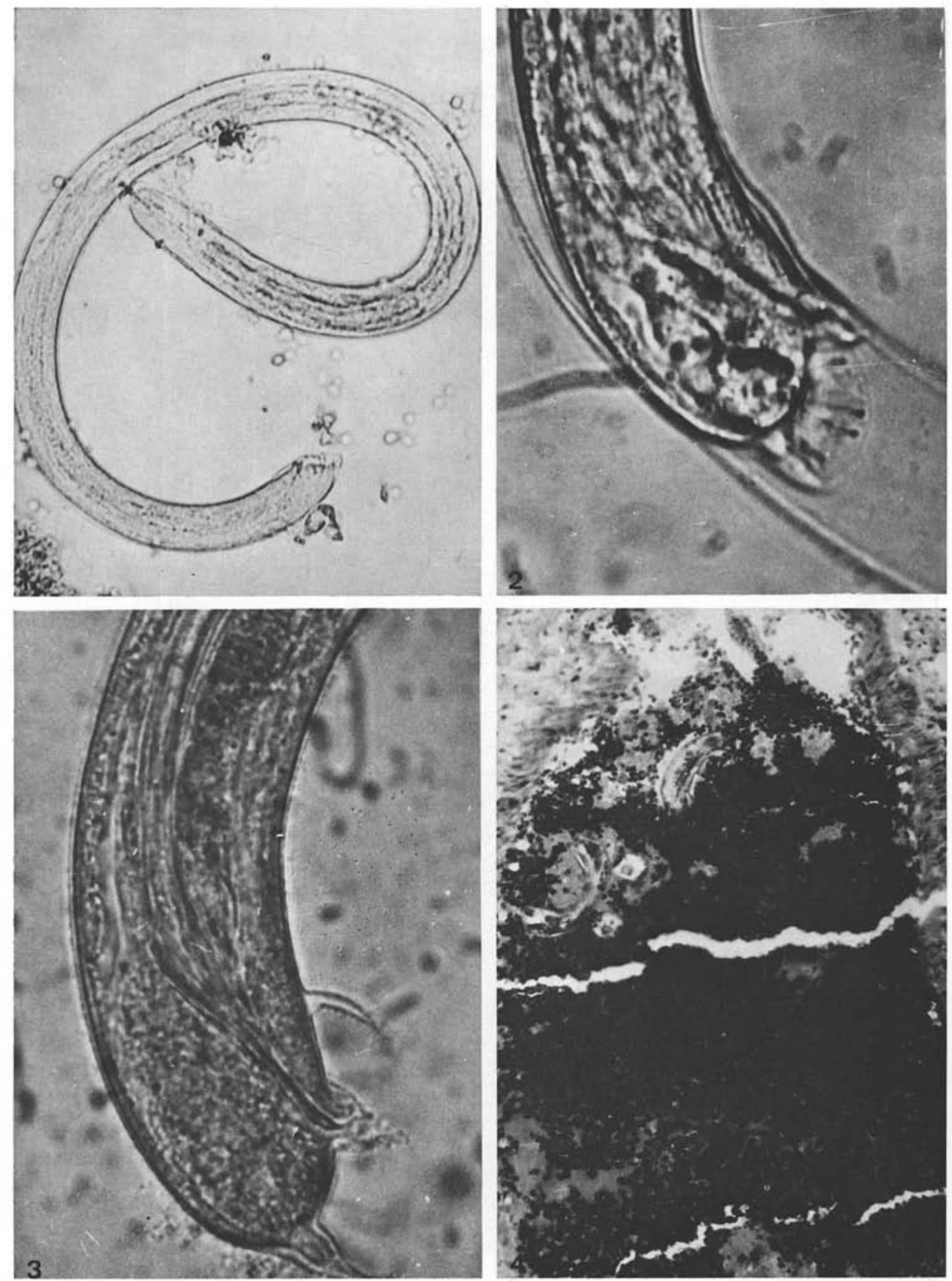

Planche III

Рното 1. - Futur mâle, au cours de la $4^{e}$ mue, extrait d'un ganglion mésentérique 5 jours après l'infestation $(\times 125)$. Рното 2. - Extrémité postérieure d'un jeune mâle au $5^{e}$ stade, encore dans son exuvie ; bourse copulatrice complètement formée $(\times 500)$. Рното 3 . - Jeune mâle au $5^{\mathrm{e}}$ stade, libéré de son exuvie, avec spicules apparents, recueilli 6 jours après l'infestation dans un ganglion mésentérique $(\times 500)$. Рното 4. - Immatures âgés de 8 jours, en coupe, dans un gros rameau de la veine porte $(\times 80)$. 

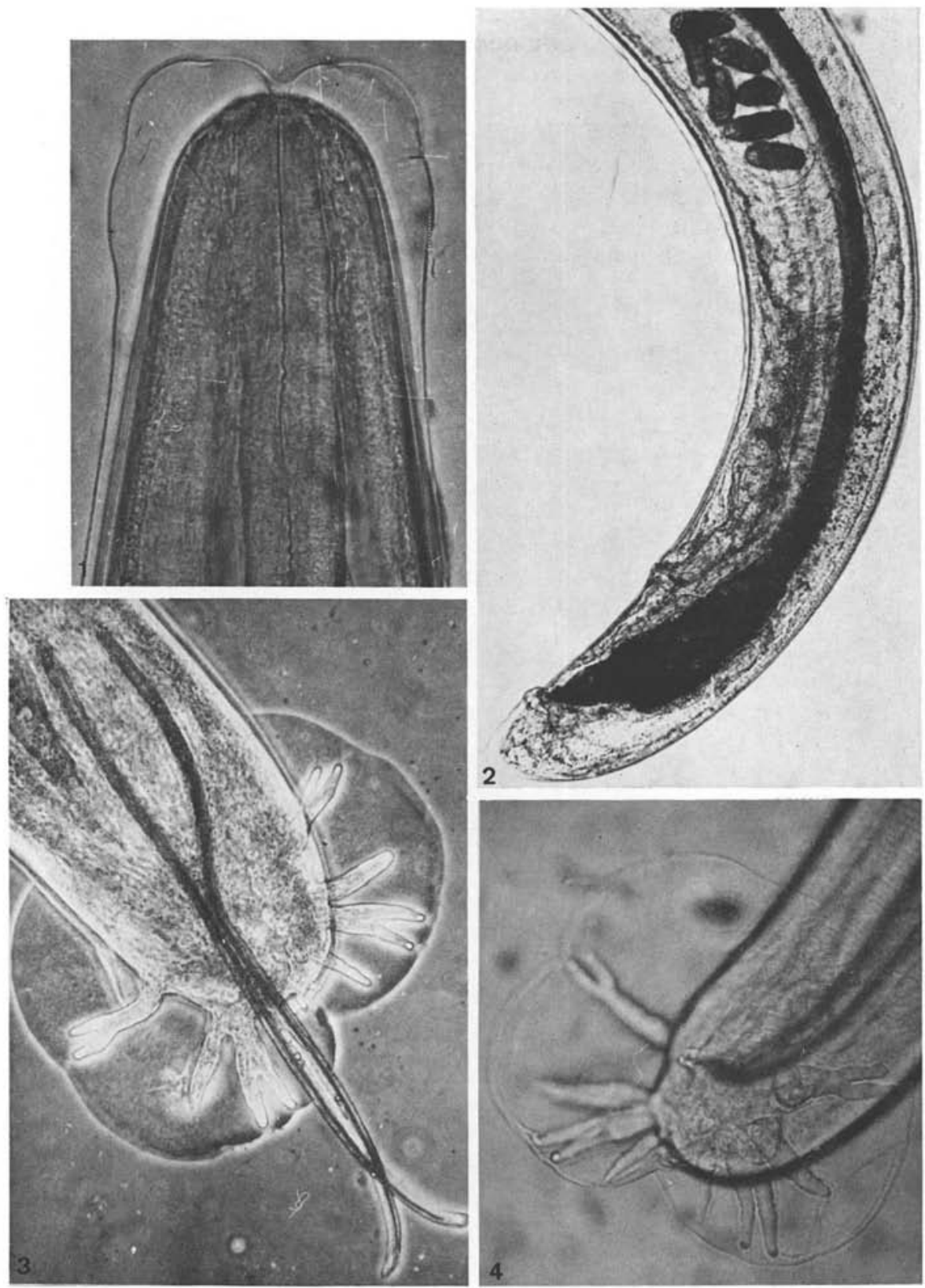

PLANCHE IV

Pното 1. - Extrémité antérieure d'une femelle adulte âgée de 37 jours montrant la gaine tégumentaire $(\times 320)$. Рното 2 . - Extrémité postérieure d'une femelle ovigère âgée de 36 jours $(\times 80)$. Рното 3. - Bourse copulatrice d'un mâle adulte, en vue ventrale $(\times 125)$. Рното 4. - Bourse copulatrice d'un male adulte, en vue latéro-ventrale montrant les papilles sensorielles de la région distale du gubernaculum $(\times 125)$. 

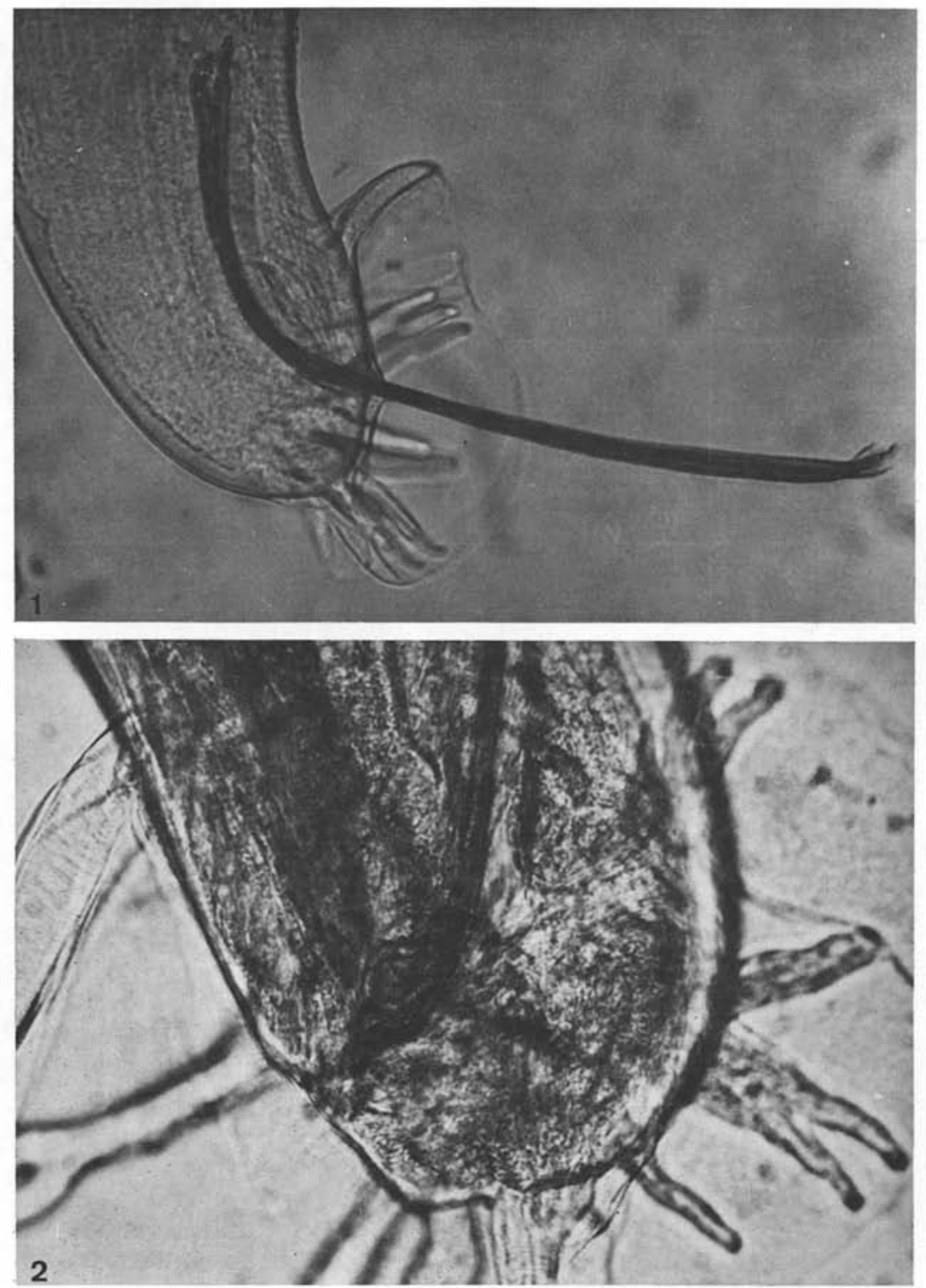

PLANCHE V

Pното 1. - Bourse copulatrice d'un mâle avec son gubernaculum apparent, en vue latérale $(\times 125)$. Pното 2. - Vue latéro-ventrale du gubernaculum $(\times 320)$. 


\section{Bibliographie}

AsH (L. R.), 1970. - Diagnostic of the third-stage larvae of Angiostrongylus cantonensis, Angiostrongylus vasorum, Aelurostrongylus abstrusus and Anafilaroïdes rostraius (Nematoda, Metastrongyloidea). J. Parasit., vol. 56, 2, 249-253.

BAILlet (C.), 1854. - Dochmius trigonocephalus (Dujardin). Histoire Naturelle des Helminthes Strongylus trigonocephalus (Rudolphi). J. Vét. du Midi, 7, 72-73.

—, 1862. - Etude comparative des caractères et de l'organisation du Dochmius trigonocephalus (Dujardin) et du ver des vaisseaux et du cœur chez le chien. J. Vét. du Midi, 25, $49-60$.

-, 1866. - Strongle des vaisseaux et du cœur du chien. Nouveau Dictionnaire prat. de Méd., chir. et d'Hyg. vétérinaire, 8, 587-588.

CENS (B.). - Evolution larvaire et migrations d'Angiostrongylus vasorum (Baillet, 1866): Nématode, Métastrongylidé dans l'organisme de son hôte définitif: le Chien. Novembre 1969 (non publiée).

Cuille (J.) et Darraspen (E.), 1930. - De la Strongylose cardio-pulmonaire du chien. Rev. Méd. vét., 39, 625-764.

— et —, 1934. - Considérations sur la strongylose cardio-pulmonaire du chien. Rev. Méd. vét. et J. Méd. vét. et de Zootechnie, 1934, 86, 481-486.

Gonçalves (P. C.), 1961. - Angiostrongylus vasorum (Baillet, 1866) novo parasito de cao no Rio Grande do Sul (Brasil). Revista da Faculdade de Agronomia e veterinaria, 4 (1), 35-40.

Guilhon (J.), 1960. - Rôle des Limacidés dans le cycle évolutif d'Angiostrongylus vasorum (Baillet, 1866). C.R. Acad. Sci., 251, 2252-2253.

-, 1963. - Rechercheз sur le cycle évolutif du Strongle des vaisseaux du chien. Bull. Acad. vét. Fr., 36, 431-442.

—, 1965. - Evolution larvaire d'Angiostrongylus vasorum dans l'organisme d'Arionidés. C.R. Acad. Sci., 261, 4225-4227.

—, 1967. - Hôtes intermédiaires et définitifs d'Angiostrongylus vasorum. C.R. I Ir Congrès latino-américain de Parasitologie, Santiago-du-Chili, p. 148.

— et de GaAlon (A.), 1969. - Evolution larvaire d'un Nématode parasite de l'appareil circulatoire du chien dans l'organisme de mollusques dulçaquicoles. C.R. Acad. Sci., $268,612-615$.

— et Afghnhi (A.), 1969. - Evolution larvaire d'Angiostrongylus vasorum (Baillet, 1866) dans l'organisme de diverses espèces de mollusques terrestres. C.R. Acad. Sci., 268, 434-436.

- et CENS (B.), 1969. - Migrations et évolution d'Angiostrongylus vasorum (Baillet, 1866) dans l'organisme du chien. C.R. Acad. Sci., 269, 2377-2380.

Laulanie (F.), 1882. - Sur une tuberculose parasitaire du chien et sur la pathogénie du follicule tuberculeux. C.R. Acad. Sci., 94, 49-52.

-, 1884. - Sur quelques affections parasitaires du poumon et leur rapport avec la tuberculose. Arch. Physiol. normale et pathologique, 3, IV, 487-511. 
Mackerras (M. J.) et Sandars (D. F.), 1955. - The life-history of the Rat lungworm Angiostrongylus cantonensis (Chen), Nematoda Metastrongylida. Aus. J. of Zoology, 3, 1-21.

Maupas (E.), 1899. - La mue et l'enkystement chez les Nématodes. Arch. de Zool. exp. et générale, 7, 563-632, Librairie Reinwald (C.), Schleicher Frères, édit.

Railliet (A.) et Cadiot (P.-J.), 1892. - Essais de transmission du Strongylus vasorum du chien au chien. C.R. Soc. Biol., 702-703.

- et HenRY (A.), 1907. - Sur les variations des strongles de l'appareil respiratoire des Mammifères. C.R. Soc. Biol., 63, 751-753.

Rosen (L.), Ash (L.) et Wallace (G. D.), 1970. - Life-history of the canine lungworm Angiostrongylus vasorum (Baillet). Am. J. Vet. Res., 31 (1), 131-143.

SERRES (E.), 1854. - Entozoaires trouvés dans l'oreille droite, le ventricule correspondant et l'artère pulmonaire d'un chien. J. vét. du Midi, 7, 72-73. 